Grossesses non planifiées et avortements à Kinshasa (République Démocratique du Congo)

Défis et progrès
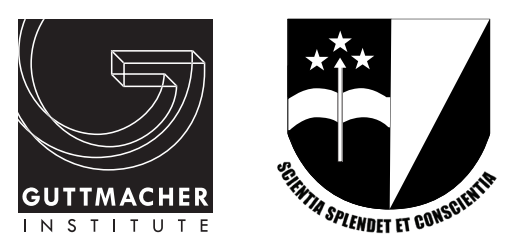



\title{
Grossesses non planifiées et avortements à Kinshasa (République Démocratique du Congo) \\ Défis et progrès
}

\author{
Naomi Lince-Deroche \\ Patrick Kayembe \\ Nakeisha Blades \\ Crispin Mabika \\ Patrice Williams \\ Susan London \\ Jesse Philbin \\ Akinrinola Bankole
}

Guttmacher Institute

Université de Kinshasa, Département des Sciences

de la Population et du Développement

École de Santé Publique de Kinshasa
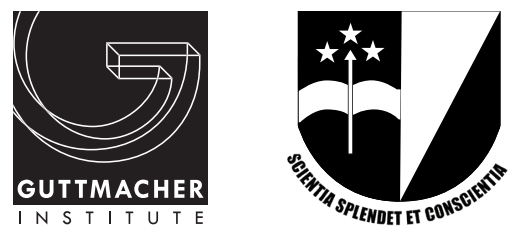


\section{Remerciements}

La rédaction de ce rapport revient à Naomi LinceDeroche, Nakeisha Blades, Patrice Williams, Jesse Philbin et Akinrinola Bankole, du Guttmacher Institute; à Patrick Kayembe et Crispin Mabika, de I'Université de Kinshasa; et à Susan London, consultante indépendante. Haley Ball, du Guttmacher Institute, en a assuré la révision.

Les auteurs tiennent à remercier leurs collègues, passées et présentes, au Guttmacher Institute pour leurs précieuses contributions: Sophia Chae, co-chercheuse principale; Ann Biddlecom, Alanna Galati, Lisa Remez, Colette Rose, Sophia Sadinsky, Susheela Singh et Elizabeth Sully, pour leur révision du rapport en cours d'élaboration; et Lorraine Kwok, pour son assistance à la recherche.

Les auteurs désirent également exprimer leur reconnaissance aux réviseurs extérieurs suivants, pour leurs retours et conseils: Jane Bertrand, à la Tulane School of Public Health and Tropical Medicine; Jacques Emina, à I'Université de Kinshasa; Kate Gilles et Jerry Parks, au Population Reference Bureau; et Françoise Mukuku, « Si Jeunesse Savait ».

Nous apprécions aussi l'apport des membres de comité consultatif: Nguma Aloïs, du Département de Gynécologie et Obstétrique à I'Université de Kinshasa; Brigitte Kini, de l'Organisation mondiale de la Santé; Lombeya Lisomba et Guy Mukumpuri, du Programme national de santé de la reproduction du ministère de la Santé (MiniSanté) en République démocratique du Congo (RDC); Alphonse Matondo, du Fonds des Nations Unies pour la population; Moïse Mbile et Mbadu Muanda, du Programme national de Santé des Adolescents au MiniSanté en
RDC; et Lisa Mahoya et Kalume Tutu, du MiniSanté en RDC.

Les données utilisées pour ce rapport sont issues de travaux de recherche effectués à Kinshasa (RDC) en 2016. Merci à Denise Ngondo, de l'École de Santé publique de I'Université de Kinshasa, et à Barthelemy Kalambayi, Francis Loka, Isaac Mukadi, Basile Tambashe et Guy Tshomba, tous du Département des Sciences de la population et du développement à l'Université de Kinshasa, pour leur contribution à l'administration, la planification et la collecte des données. Nous sommes enfin reconnaissants à l'endroit des enquêteurs, chefs d'équipes et répondants et répondantes pour leur contribution et leur participation à l'étude.

Lélaboration de ce rapport a bénéficié de subventions de la Fondation David and Lucile Packard, de l'Agence Suédoise de Coopération au Développement International, du Ministère Néerlandais des Affaires Etrangères et de l'Agence Norvégienne de Coopération au Développement, ainsi que d'UK Aid sous les auspices du gouvernement britannique. Les observations et les conclusions exprimées dans ce rapport sont celles des auteurs. Elles ne reflètent pas nécessairement les positions ou les politiques des bailleurs de fonds. Le Guttmacher Institute tient à exprimer sa profonde gratitude pour le financement sans restriction dont il bénéficie de la part de nombreux particuliers et fondations - notamment les importantes contributions de la Fondation William and Flora Hewlett et de la Fondation David and Lucile Packard, qui sous-tendent la totalité de son travail. 


\section{Table des matières}

Résumé

Contexte de la vie sexuelle et reproductive des femmes

Incidence et pratique de

I'avortement

10

Conséquences sanitaires de I'avortement non médicalisé et soins après avortement

15

4 Facteurs contribuant à

l'avortement non médicalisé

20

Conclusions et recommandations

25

Références

4

6

4 


\section{Résumé}

Le code pénal de la République Démocratique du Congo (RDC) interdit l'avortement, et bien qu'un article du code de déontologie des médecins l'admette dans de très strictes circonstances, les services médicalisés restent largement inaccessibles. La publication récente du Protocole de Maputo dans le journal officiel de la nation pourrait être un signe d'une tendance à la libéralisation du statut légal de l'avortement, mais l'application du protocole ne se fera pas du jour au lendemain. Aussi la grande majorité des femmes de Kinshasa qui choisissent d'interrompre une grossesse non planifiée continuera de le faire dans la clandestinité — souvent dans des conditions non médicalisées susceptibles de mettre en péril leur santé, voire leur vie.

\section{L'avortement est fréquent dans la capitale de la RDC}

- L'Étude de 2016 sur l'avortement à Kinshasa, menée par le Guttmacher Institute en collaboration avec le Département des Sciences de la population et du développement et l'École de Santé publique de l'Université de Kinshasa, est la première qui ait cherché à estimer l'incidence de l'avortement à Kinshasa.

- On estime à 146700 le nombre d'avortements ayant eu lieu à Kinshasa en 2016. Le taux d'avortement correspondant - 56 interruptions pour 1000 femmes âgées de 15 à 49 ans — est inférieur à ceux relevés parmi les femmes de même âge ou d'âge comparable dans d'autres grandes villes d'Afrique subsaharienne, notamment Addis Abeba (Éthiopie; 92 pour 1 000), Kampala (Ouganda; 77) et Kigali (Rwanda; 87). II est cependant supérieur à ceux enregistrés à Nairobi (Kenya; 32) et Dakar (Sénégal; 21).

- Parmi les patientes en soins après avortement qui ont déclaré avoir tenté d'interrompre leur grossesse, la méthode abortive la plus couramment utilisée était le misoprostol. Plus de la moitié des femmes (56\%) ont déclaré avoir utilisé le misoprostol et près de la moitié des femmes de ce groupe l'avaient utilisé en combinaison avec une autre méthode.

\section{L'avortement à Kinshasa s'accompagne souvent de conséquences de santé graves}

- En 2016, selon les estimations, 37870 Kinoises auraient reçu des soins pour complication suite à un avortement.

- De l'avis des informateurs clés qui sont au courant sur ce qui se passe en matière d'avortements à Kinshasa, 34\% des femmes qui interrompent une grossesse présentent vraisemblablement des complications suffisamment graves pour justifier un traitement dans une formation sanitaire. Dans ce groupe de femmes, estiment-ils, $77 \%$ obtiennent les soins dont elles ont besoin et $23 \%$ se font soigner en dehors des formations sanitaires ou pas du tout.

- Sur l'ensemble des patientes qui ayant reçu les soins après un avortement, $5 \%$ ne présentaient aucun signe de complications, 33\% présen taient de légères complications, $46 \%$, des complications de gravité moyenne et $16 \%$, des complications graves telles que l'état de choc, la défaillance d'organe, la péritonite généralisée ou la mort. Le taux de traitement après avortement a été estimé à 14 pour 1000 femmes âgées de 15 à 49 ans.

- Presque tous les services de soins après avortement à Kinshasa (93\%) sont obtenus dans le secteur privé, où les services sont probablement plus onéreux que dans les formations sanitaires de l'État. Il peut s'agir là d'un obstacle pour les femmes qui ont besoin de ces services, en particulier celles défavorisées sur le plan socio-économique.

- Parmi toutes les femmes qui ont avorté, les femmes à faible revenu se sont révélées deux fois plus susceptibles de présenter des complications 
de gravité moyenne ou graves, par rapport aux femmes au revenu plus élevé. Il semble aussi que les femmes pauvres soient moins susceptibles d'obtenir des soins après avortement.

\section{La grossesse non planifiée est fréquente à Kinshasa}

- Sur le nombre total de grossesses estimé à 563100 à Kinshasa en 2016, 61\% n'étaient pas planifiées, soit un taux de 147 grossesses non planifiées pour 1000 femmes âgées de 15 à 49 ans.

- Les données de l'Enquête démographique et de santé (EDS) indiquent que les Kinoises ont du mal à atteindre leurs objectifs de fécondité: en 2013-2014, les femmes désiraient en moyenne 3,6 enfants mais elles en avaient 4,2. En 2017, selon l'initiative Performance Monitoring and Accountability (PMA) 2020, le besoin non satisfait de contraception était assez élevé: un quart des femmes mariées éprouvaient un besoin non satisfait de planification familiale.

- Les obstacles à la pratique contraceptive à Kinshasa tiennent aux caractéristiques individuelles des femmes - y compris le jeune âge et le faible pouvoir socioéconomique - ainsi que des difficultés systémiques rencontrées, notamment le manque de sensibilisation à la contraception et le choix limité des méthodes proposées dans les formations sanitaires.

- Environ $25 \%$ des femmes qui ont subi des violences physique pendant la grossesse pour lesquelles elles ont eu besoin de soins après l'avortement ont indiqué que cette violence avait conduit à l'avortement. Parmi les femmes ayant déclaré avoir subi des rapports sexuels forcés, $30 \%$ ont indiqué que la grossesse à propos de laquelle elles étaient venues se faire soigner était le résultat de cette violence.

\section{Des mesures doivent être prises pour} réduire les grossesses non planifiées et de I'avortement non médicalisé en RDC

- La poursuite des efforts entrepris récemment pour améliorer l'accès à la contraception et son adoption aidera les femmes à éviter les grossesses non planifiées et l'avortement non médicalisé. Ces efforts doivent viser une meilleure qualité et la disponibilité constante d'une large gamme de méthodes dans les formations sanitaires du secteur publique, tout en assurant l'offre d'options économiquement abordables et de qualité acceptable. Les services doivent être adaptés aux besoins particuliers des femmes jeunes et les communautés doivent être informées sur les méthodes disponibles.

- Les autres causes fondamentales de la grossesse non planifiée et de l'avortement, comme l'inégalité de genre et les violences faites aux femmes, doivent aussi être résolues.

- L'adoption récente du Protocole de Maputo laisse envisager la baisse du nombre de grossesses non planifiées et d'avortements non médicalisés en RDC. Sa mise en œuvre, qui appelle les pays signataires à autoriser l'avortement dans de nombreuses circonstances, demandera un effort considérable, notamment en termes de révision du code pénal; de formation des professionnels de santé (y compris le personnel infirmier et les sages-femmes) et de leur dotation en équipements et intrants leur permettant d'assurer les avortements médicalisés; ainsi que l'information de la population concernant l'accès aux services. Le passage à la légalité exigera aussi une déstigmatisation, en sensibilisant notamment les prestataires de santé et autres intervenants à la nécessité pour les femmes de bénéficier de soins médicalisés, sans jugement.

- La transition sera probablement longue vers la pleine prestation de services d'avortement médicalisé. Aussi la capacité du système sanitaire de Kinshasa - tant au niveau primaire que d'orientation - doit-elle être renforcée pour assurer la fourniture de soins après avortement conformes aux normes internationales. Le personnel infirmier et les sages-femmes devront être formés à la pratique médicalisée de l'AMIU et à l'utilisation du misoprostol pour l'avortement incomplet sans complications, comme recommandé par les directives applicables.

- Il serait aussi utile de rendre les services de soins après avortement plus abordables pour que les femmes qui en ont besoin soient en mesure de les obtenir. 


\section{(1) Contexte de la vie sexuelle et reproductive des femmes}

Le code pénal de la République Démocratique du Congo (RDC) interdit l'avortement dans toutes les circonstances ${ }^{1}$ et, bien qu'il soit depuis longtemps officieusement toléré, l'accès à des services médicalisés reste extrêmement limité. Un article du code de déontologie médicale admet l'avortement pour sauver la vie d'une femme enceinte (pourvu que l'interruption de la grossesse soit pratiquée par un médecin, avec l'approbation de deux autres médecins) ${ }^{2}$, mais il semble que relativement peu de femmes puissent obtenir un avortement sûr par cette voie. La majorité des interruptions de grossesse sont par conséquent clandestines et donc potentiellement risquées. Le statut illégal de l'avortement fait aussi qu'il est extrêmement difficile de le suivre ou de le mesurer dans le pays et que I'on ne peut que spéculer, depuis de nombreuses années, quant au taux de l'avortement clandestin en RDC et dans la capitale.

Cela dit, la publication historique du Protocole à la Charte africaine des droits de l'Homme et des peuples relatif aux droits de la femme en Afrique, également connu sous le nom de Protocole de Maputo, dans le journal officiel de la nation congolaise en 2018 pourrait marquer une nouvelle ouverture de la part du gouvernement de la RDC sur la question de la libéralisation de la loi sur l'avortement ${ }^{3}$. Décrit comme «le principal instrument juridique du continent sur les droits des femmes ${ }^{4} »$, le Protocole stipule que les États signataires protégeront le droit des femmes à avorter, à contrôler leur fécondité et à adopter la méthode de contraception de leur choix, entre autres droits relatifs à leur santé. Il enjoint par ailleurs les États d'autoriser l'avortement dans les cas d'agression sexuelle, de viol, d'inceste ou d'anomalie fœtale grave, ou lorsque la vie ou la santé physique ou mentale de la femme est en danger. Il oblige également les signataires à protéger les autres droits de santé sexuelle et reproductive.

En publiant le Protocole de Maputo, le gouvernement de la RDC a officiellement donné force de loi au traité dans le pays, signalant sa prise de distance par rapport à l'interdiction totale de l'avortement en RDC. Des efforts devront être consentis pour harmoniser le code pénal du pays avec le Protocole de Maputo en vue d'établir la légalité claire et constante de l'avortement et de mettre en place les mécanismes qui assureront que les femmes légalement autorisées à se faire avorter aient accès au service. II faudra un certain temps avant que l'accès aux services d'avortement médicalisé devienne réalité en RDC. En attendant, la grande majorité des femmes désireuses d'interrompre une grossesse non planifiée continueront à le faire clandestinement parfois au moyen de méthodes non médicalisées, avec l'aide de prestataires non qualifiés ou les deux.

Il importe entre-temps de cerner la mesure de l'avortement en RDC. À ce jour, la recherche ne s'est guère penchée sur les circonstances et les conséquences de l'avortement non médicalisé chez les femmes qui ne peuvent pas interrompre leur grossesse dans de bonnes conditions de sécurité. Considérant aussi la grande taille du pays, il est du reste extrêmement difficile d'y mener une recherche au niveau national. Ce rapport constitue une première démarche pour combler ce manque d'information. II résume les résultats de l'Étude de 2016 sur l'avortement à Kinshasa (voir l'encadré, page 7), première en son genre à estimer l'incidence de l'avortement dans la capitale congolaise. Outre la couverture de l'incidence de l'avortement à Kinshasa, le rapport:

- donne une estimation de l'incidence de la grossesse non planifiée

- documente l'ampleur et la gravité de la morbidité liée à l'avortement et

- élucide les facteurs qui contribuent au risque d'obtenir un avortement non médicalisé en décrivant les caractéristiques des patientes traitées dans les formations sanitaires pour cause de complications d'un avortement.

La concentration sur la ville de Kinshasa ne doit pas être interprétée comme impliquant que le problème de l'avortement non médicalisé ne concerne 
que la capitale. Dans la mesure du possible, les résultats de la recherche et leurs implications en termes d'élaboration des politiques et de planification des programmes sont considérés non seulement pour Kinshasa, mais pour la RDC dans son ensemble.

\section{Contexte de la ville et du pays}

Kinshasa est la deuxième ville la plus peuplée $d^{\prime}$ Afrique subsaharienne ${ }^{11,12}$. En 2014, elle comptait quelque 11 millions d'habitants, soit 13\% de la population totale de la RDC. La population kinoise enregistre une croissance galopante depuis quelques années - dans une mesure moyenne de 4,2\% par an entre 2010 et 2015, supérieure au taux de croissance démographique congolais global de 3,3\% entre 2009 et $2016^{12,13}$. Les conditions socioéconomiques sont meilleures à Kinshasa, par rapport au reste du pays, bien que le niveau général de pauvreté reste élevé ${ }^{14}$.

Dans ce rapport, les données de Kinshasa sont replacées dans le contexte national. Le pays rencontrera d'importants défis de développement au cours de ses efforts en vue de l'amélioration de l'accès à des services d'avortement médicalisé. Environ trois quarts $(77 \%)$ de la population congolaise vit en-dessous du seuil international de pauvreté fixé à 1,90 dollar américain par jour ${ }^{15}$. L'indice de développement humain, qui mesure trois dimensions essentielles du développement humain (une vie longue et en bonne santé, l'accès à la connaissance et le niveau de vie), classe la RDC au 176 rang sur 189 nations, dans la catégorie de « faible développement humain ${ }^{16} »$. Les femmes de la RDC en subissent les inconvénients de manière disproportionnée. Elles sont moins scolarisées et gagnent moins que leurs homologues masculins, atteignant dès lors un score inférieur à celui des hommes sur les deux dernières dimensions du développement humain.

Dans de nombreux contextes africains, les indicateurs de santé et de développement - y compris ceux de santé reproductive - sont souvent meilleurs dans les capitales que dans les pays pris dans leur ensemble. D'après les résultats de la dernière Enquête démographique et de santé (EDS) menée en RDC en 2013-2014, les données relatives aux femmes âgées de 25 à 49 ans indiquent un âge médian au moment du mariage de 23 ans à Kinshasa, par rapport à 19 ans à l'échelle nationale 9 . L'âge médian au premier rapport sexuel, parmi les femmes âgées de 20 à 49 ans, était de 18 ans à Kinshasa, soit un peu plus d'un an de plus que la

\section{Source des données}

L'Étude de 2016 sur l'avortement à Kinshasa est le produit d'un effort conjoint de Guttmacher Institute, du Département des Sciences de la population et du développement et de l'École de Santé publique de I'Université de Kinshasa. L'équipe de recherche a mené des enquêtes sur les pratiques abortives et sur les services de soins après avortement auprès de trois groupes: des représentants de formation sanitaire, des informateurs clés au courant de la situation et des patientes en soins après avortement. La méthodologie pour chaque enquête est résumée plus bas. Les méthodes et les résultats de ces travaux ont aussi été publiés en détails dans deux articles cités tout au long du rapport ${ }^{5,6}$.

- Enquête dans les formations sanitaires. Cette enquête a été menée à Kinshasa, auprès d'un échantillon représentatif composé de 361 hôpitaux et centres de santé publics et privés qui traitent les femmes souffrant de complications liées à l'avortement. Dans chaque formation, un membre du personnel de haut niveau ou un administrateur au courant des services de soins après avortement assurés dans son établissement a été invité à participer. Dans les hôpitaux, les répondants étaient généralement des obstétriciens-gynécologues ou le responsable du service d'obstétrique et gynécologie. Dans les centres de santé, il s'agissait généralement du directeur de l'établissement ou d'un prestataire de soins, tel qu'une sage-femme ou une infirmière. Les données concernant le nombre de femmes ayant obtenu des soins après avortement dans chaque formation en 2016 sont pondérées pour produire une estimation à l'échelle de la ville.

- Sondage des professionnels de la santé. Pour cette enquête, 115 professionnels de la santé, chercheurs, décideurs politiques, acteurs de plaidoyer, assistants sociaux, membres du personnel d'ONG et autres personnes informées, sélectionnés à dessein pour leur connaissance de la prestation de l'avortement et des soins après avortement à Kinshasa, ont été interviewés. Les participants ont été invités à donner leur opinion sur les types de prestataires auxquels les Kinoises s'adressent pour se faire avorter, ainsi que sur les méthodes abortives pratiquées, la probabilité de complications, la proportion des femmes présentant des complications après l'avortement et la proportion de ces dernières qui viennent se faire soigner dans une formation sanitaire. II leur a été demandé de répondre séparément pour les femmes pauvres et pour celles non pauvres, sans leur donner toutefois de définition claire de la notion de " pauvre".

- Enquête prospective de morbidité. Cette enquête repose sur des entretiens avec un échantillon probabiliste de 867 femmes hospitalisées ou en soins ambulatoires ayant reçu des services de soins après avortement suite à un avortement non médicalisé ou à une fausse couche, dans un échantillon représentatif des formations sanitaires comprises dans l'Enquête dans les formations sanitaires. Les participantes ont été interrogées sur leurs antécédents génésiques, leurs caractéristiques socioéconomiques et démographiques et leur expérience de l'avortement ou de la fausse couche. Pour les femmes qui y ont consenti, une information complémentaire de diagnostic clinique et traitement a été obtenue sur la base de leur dossier médical et auprès de leur prestataire de soins. Le statut de pauvreté a été indexé au moyen de questions relatives aux possessions du ménage, selon l'approche généralement adoptée dans I'EDS ${ }^{78}$. L'index a ensuite été standardisé en fonction de la distribution relative à Kinshasa dans I'EDS 2013-2014, car les femmes traitées pour complications d'un avortement étaient considérées non représentatives de l'ensemble des femmes en âge de procréer.

Dans tout le rapport, les données de l'Étude sur l'avortement à Kinshasa sont complétées de celles, au niveau de la ville et du pays, de l'enquête PMA 2020 menée en 2017 et de l'EDS 2013-2014,10. 


\section{Définitions}

Les définitions adoptées dans la réalisation de ce rapport sont celles utilisées dans I'Enquête démographique et de santé.

- Les méthodes contraceptives modernes comprennent la stérilisation féminine et masculine, la pilule contraceptive, le stérilet (DIU), l'injectable, l'implant, le préservatif féminin et masculin, le diaphragme, la mousse et le gel contraceptifs, la méthode de l'aménorrhée lactationnelle, la méthode "Standard Days ", la cape cervicale et l'éponge contraceptive.

- Les méthodes contraceptives traditionnelles comprennent I'abstinence périodique (méthode du rythme ou Ogino et méthode du calendrier), le retrait et les méthodes populaires et spirituelles.

- Les femmes qui présentent un besoin non satisfait de contraception sont celles, âgées de 15 à 49 ans, qui sont fécondes mais qui désirent éviter une grossesse sans toutefois pratiquer une méthode de contraception.

- Les grossesses non planifiées sont celles non désirées ou inopportunes au moment de la conception. médiane nationale de 16,8 ans. Parmi les femmes âgées de 25 à 49 ans, l'âge médian à la première maternité était de 22 ans dans la capitale, par rapport à 20 ans sur l'ensemble du pays. Plus frappant peut-être, la proportion des jeunes femmes de 15 à 19 ans qui avaient déjà accouché ou qui étaient enceintes au moment de l'EDS était de 13\% à Kinshasa, par rapport à $27 \%$ à l'échelle nationale.

Près de neuf femmes sur 10 âgées de 15 à 49 ans en RCD déclaraient en 2013-2014 avoir entendu parler de la contraception moderne (voir l'encadré) ${ }^{9}$. Huit pour cent seulement en pratiquaient toutefois les méthodes à l'échelle nationale. La proportion était plus grande parmi les femmes mariées de Kinshasa (19\%). Sur l'ensemble de la RDC, les femmes déclaraient désirer un total moyen de 5,7 enfants, alors que les Kinoises n'en désiraient que 3,6. Pourtant, compte tenu de l'indice synthétique national de fécondité calculé à 4,2, les femmes de la capitale avaient plus d'enfants qu'elles n'en désiraient en 2013-2014. Ensemble, ces indicateurs signalent l'existence d'un besoin important de contraception non satisfait dans la capitale. Autrement dit, de grandes proportions de Kinoises sont fécondes et désirent éviter une grossesse pendant au moins deux ans mais ne pratiquent pas la contraception. À Kinshasa en 2013-2014, 23\% des femmes mariées ou en union, $31 \%$ des femmes célibataires sexuellement actives et $16 \%$ de la totalité des femmes répondaient à la définition d'un besoin non satisfait de contraception.

En RDC, les défis posés aux femmes en termes de contrôle de leur fécondité se trouvent exacerbés par les niveaux extrêmement élevés de la violence à leur encontre. À partir du début des années 1990, le pays a sombré dans deux décennies d'instabilité politique et de conflit armé (principalement centré dans les régions de l'est) ${ }^{17,18}$. La violence sexuelle, souvent érigée en tactique de guerre durant ces années de conflit, demeure courante. En 2011, d'après les données de l'EDS 2007, les chercheurs ont estimé, à l'échelle nationale, le nombre de femmes violées et victimes de violences sexuelles perpétrées par un partenaire intime. Leurs résultats révèlent que 1,7 à 1,8 million de femmes en RDC auraient été violées durant leur vie, et plus de 400000 l'auraient été durant les 12 derniers mois - soit quatre femmes violées toutes les cinq minutes dans le pays ${ }^{19}$. Plus de trois millions de femmes, ou 35\% de la population féminine âgée de 15 à 49 ans en RDC, ont déclaré avoir subi des violences sexuelles aux mains d'un partenaire intime. Par contre, la proportion des femmes âgées de 20 à 44 ans qui déclarent de telles violences était de $12 \%$ au Rwanda en 2005 , $12 \%$ au Zimbabwe en $2005-2006$ et $13 \%$ au Malawi en $2004^{20}$

La dernière EDS apporte une information mise à jour sur les taux de violence physique et sexuelle à l'encontre des femmes. À Kinshasa en 2013-2014, $57 \%$ des femmes de 15 à 49 ans déclaraient avoir subi une violence physique au moins une fois depuis l'âge de 15 ans, perpétrée dans la majorité des cas par leur partenaire actuel ou ancien. De plus, $21 \%$ de ces femmes déclaraient avoir subi de telles violences " parfois ou fréquemment » au cours des 12 derniers mois. La proportion de femmes déclarant avoir subi des violences sexuelles atteint $27 \%$ sur l'ensemble de la RDC et $16 \%$ à Kinshasa, et la proportion de celles ayant subi cette violence au cours des 12 derniers mois est de $16 \%$ et $6 \%$, respectivement ${ }^{9}$. Malgré I'amélioration que révèlent ces valeurs par rapport aux résultats de 2007, le niveau de violence sexuelle dans le pays reste élevé. La violence, sexuelle en particulier, peut exposer les femmes à de nombreux risques de santé.

La précocité de l'activité sexuelle, les faibles niveaux de pratique contraceptive et l'expérience de la violence comptent au nombre des facteurs susceptibles de contribuer au risque de grossesse et de maternité non planifiées parmi les femmes de Kinshasa9. Les rares travaux de recherche qui se sont penchés sur l'avortement en RDC dans le passé indiquent que les femmes se font avorter pour atteindre leurs objectifs de fécondité21-23. 
Une enquête sur les ménages menée à Kinshasa en 1990 a révélé que $15 \%$ des femmes qui avaient déjà été enceintes s'étaient fait avorter au moins une fois ${ }^{21}$ et une autre, en 2006, que $16 \%$ des femmes qui avaient déjà été sexuellement actives déclaraient de même au moins un avortement ${ }^{24}$. Les études indiquaient aussi la prévalence de l'avortement, en particulier parmi les adolescentes. Des entretiens tenus en 2009-2010 avec des adolescentes résidentes de trois zones périphériques de Kinshasa ont révélé que $60 \%$ des 14 à 20 ans s'étaient précédemment avortées elles-mêmes clandestinement ${ }^{22}$. Les répondantes ont indiqué avoir été motivées à recourir à l'avortement de peur qu'une grossesse hors mariage menée à terme ne les couvre d'opprobre et n'anéantisse leurs perspectives de mariage, invoquant aussi leur incapacité économique à subvenir aux besoins d'un enfant comme raison de leur choix d'interrompre la grossesse.

\section{Priorisation de la santé sexuelle et reproductive en dépit des contraintes du système sanitaire} L'instabilité prolongée en RDC a affecté les indicateurs macroéconomiques du pays, en amplifiant l'inégalité, en ralentissant la croissance économique et en épuisant les ressources ${ }^{16,25,26}$. En 2001, la Déclaration d'Abuja engageait les nations d'Afrique à consacrer $15 \%$ de leur budget national à la santé ${ }^{27}$ dès l'année 2015. En 2009 pourtant, les dépenses de la RDC à ce poste, par rapport au budget national, étaient équivalentes à celles de l'année 2000. Bien que la RDC n'ait pas atteint les objectifs de la Déclaration d'Abuja, des progrès ont toutefois été enregistrés: entre 2011 et 2015 , les dépenses de santé, en proportion du budget national, sont passées de 3,4\% à 8,6\%, et 2015 a marqué la première année au cours de laquelle le gouvernement a prévu un financement distinct pour les médicaments de base et les contraceptifs ${ }^{28}$.

Le sous-financement des systèmes sanitaires donne généralement lieu à un accès limité aux services de santé et à de piètres résultats de santé. $\mathrm{En} \mathrm{RDC}$, ce sous-financement, rajouté à l'instabilité politique, s'est avéré particulièrement préjudiciable à la santé sexuelle et reproductive. Le pays avait bien un programme de planification familiale entretenu par les dons de bailleurs dans les années 1980, mais ce soutien s'est éteint avec l'enracinement du conflit, sans reprendre avant l'année 2005 environ ${ }^{18}$. Aujourd'hui, l'immensité du pays et son infrastructure inadéquate, au niveau des transports notamment, viennent renforcer la précarité des résultats sanitaires. En 2015, le taux de mortalité maternelle estimé était de $27 \%$ supérieur en RDC à la moyenne de l'Afrique subsaharienne, soit 693 décès maternels pour 100000 naissances vivantes, contre 546 pour la région ${ }^{29}$.

Heureusement, le gouvernement actuel de la RDC a fait de l'amélioration des indicateurs de santé sexuelle et reproductive une priorité. En 2014, le pays a adopté son Plan stratégique national à vision multisectorielle de la planification familiale ${ }^{30}$. Les six objectifs du plan sont: obtenir l'engagement effectif du gouvernement en faveur de la planification familiale, améliorer l'accès des hommes et des femmes aux services de planification familiale dans le secteur public et dans le secteur privé, augmenter la qualité des services, stimuler la demande de la planification familiale, élaborer et renforcer un système efficace de gestion logistique des contraceptifs et mettre en place un système fiable d'évaluation des résultats. La RDC a pris des engagements analogues en vertu de l'initiative FP2020. En 2013, le pays s'est engagé à faire passer la prévalence contraceptive à 19\% (en partant de 5,4\%) à l'horizon $2020^{31}$, affectant par ailleurs une ligne budgétaire aux contraceptifs, sous allocation annuelle d'un million de dollars. En 2017, cette allocation est passée à 2,5 millions de dollars par $\mathrm{an}^{32}$. Plus généralement, le pays s'est engagé à faire voter une nouvelle loi en faveur de la santé sexuelle et reproductive à l'échelle nationale, ainsi qu'à amender les lois existantes pour « protéger les adolescentes contre le mariage précoce en favorisant l'éducation, la sensibilisation, l'intégration sociale, et les programmes d'autonomisation des femmes ${ }^{32(p .2)} »$.

Enfin, le gouvernement de la RDC a, avec la publication du Protocole de Maputo, fait un grand pas en faveur d'un meilleur accès aux services de santé sexuelle et reproductive - y compris les services d'avortement médicalisé. À l'heure où le pays se met à harmoniser ses lois et à prendre des mesures propices à l'accès à des services d'avortement sûr, il importe d'examiner les pratiques abortives actuelles pour mieux définir les priorités. 


\section{(2) Incidence et pratique de l'avortement}

Les données de l'Enquête dans les formations sanitaires et du Sondage des professionnels de la santé réalisés dans le cadre de l'Étude de 2016 sur l'avortement à Kinshasa indiquent un nombre approximatif de 146700 avortements pratiqués à Kinshasa en $2016^{5}$. Cela correspond à un taux de 56 avortements pour 1000 femmes âgées de 15 à 49 ans et de 44 avortements pour 100 naissances vivantes, soit un peu plus de deux interruptions de grossesse pour cinq naissances vivantes. Une étude publiée peu après celle de 2016 sur l'avortement à Kinshasa corrobore ces constats. Les chercheurs de cette seconde étude ont suivi une approche différente, celle de «la confidente», selon laquelle les femmes sont interrogées sur les avortements obtenus par d'autres qui se confient à elles. Le taux d'avortement résultant en était de 55 pour 1000 femmes âgées de 15 à 49 ans $^{33}$.

Le taux d'avortement à Kinshasa est inférieur à ceux relevés dans d'autres capitales d'Afrique subsaharienne, comme Addis Abeba (92 pour 1000 femmes) ${ }^{34}$, Kampala $(77)^{35}$ et Kigali $(87)^{36}$, mais supérieur au taux combiné de Nairobi et de la région Centre du Kenya (32) ${ }^{37}$ et à celui de Dakar (21) ${ }^{38}$. Le taux global pour la RDC dans son ensemble est inconnu, mais les taux sont souvent plus élevés dans les capitales, par rapport aux zones moins peuplées. Quelques raisons expliquent cette divergence. D'abord, les zones urbaines offrent typiquement un meilleur accès aux services de santé en général et les habitantes des zones rurales se rendent dans les villes pour obtenir les services requis ${ }^{34}$. Les populations urbaines tendent aussi à désirer moins d'enfants que celles des milieux ruraux ${ }^{5}$. Enfin, les opinions sociales relativement libérales généralement caractéristiques des villes peuvent s'étendre aux normes sociales plus ouvertes à l'avortement.

\section{Pratique de l'avortement à Kinshasa}

La recherche antérieure a montré que les Congolaises peuvent tenter de provoquer un avortement selon les méthodes traditionnelles, à l'aide par exemple de « cimpokolo » et d'autres herbes, de quinine ou d'autres médicaments oraux obtenus auprès d'amies, de membres de la famille ou de praticiens traditionnels $s^{39,40}$. On sait aussi que les prestataires et les femmes enceintes interrompent clandestinement les grossesses au moyen de misoprostol ${ }^{41}$. Ce médicament, homologué dans le pays sous la marque Cytotec pour le traitement des ulcères gastriques, offre un moyen sûr et efficace de provoquer un avortement quand il est administré sous conseil approprié, mais l'accès n'y est pas encore généralisé.

Lors du Sondage des professionnels de la santé effectué dans le cadre de l'Étude de 2016 sur l'avortement à Kinshasa, les informateurs clés au courant de la santé sexuelle et reproductive, y compris des professionnels de la santé, des administrateurs de formation sanitaire, des éducateurs et des militants de la sensibilisation communautaire, ont exprimé leurs opinions sur les méthodes utilisées pour interrompre la grossesse ${ }^{42}$. Selon les entretiens réalisés pour ce sondage, ces méthodes sont nombreuses à Kinshasa. Au moins neuf informateurs clés sur 10 se rejoignent pour dire que la dilatation et curetage (DC) et les surdoses de produits pharmaceutiques tels que la quinine, les antiparasitaires et les antibiotiques sont utilisés. L'administration orale et vaginale de misoprostol est signalée par environ quatre cinquièmes des informateurs. De nettes majorités font aussi état de l'aspiration intrautérine manuelle ou électrique et des méthodes traditionnelles (comme le recours à des remèdes oraux et vaginaux à base de plantes). Environ la moitié des informateurs indiquent que l'avortement est parfois provoqué au moyen d'objets tranchants ou pointus (tels que des manches de balai ou des bâtons), tandis que deux répondants sur cinq mentionnent la prise orale de médicaments hormonaux ou l'injection de quinine ou d'oxytocine, notamment. 
De l'avis des informateurs clés, la méthode utilisée dépend du prestataire ou de la source. Les médecins, les cliniciens et les infirmières pratiquent le plus couramment la méthode $\mathrm{DC}$, tandis que les praticiens traditionnels administrent plus communément des herbes ou décoctions par voie orale et que les pharmaciens proposent le misoprostol. Les femmes enceintes qui cherchent à provoquer elles-mêmes leur avortement semblent recourir principalement au misoprostol et les informateurs font remarquer à ce sujet que la majorité des femmes qui utilisent le misoprostol l'obtiennent de formations sanitaires publiques ou privées, au domicile privé de prestataires ou en pharmacie. Enfin, les autres prestataires non qualifiés sembleraient procéder le plus souvent par introduction d'objets tranchants ou pointus dans le vagin pour provoquer l'avortement.

L'Organisation mondiale de la Santé recommande l'avortement médicamenteux par administration d'une combinaison de misoprostol et de mifépristone (ou de misoprostol seul en l'absence de disponibilité de mifépristone, tel que cela est le cas en RDC) ou l'avortement chirurgical par aspiration intra-utérine pour les interventions pratiquées durant le premier trimestre (ainsi que pour la plupart de celles plus tardives) ${ }^{43}$. Elle ne recommande plus la méthode DC à aucun moment de la gestation, car celle-ci est plus lente, plus douloureuse et moins sûre que les autres approches. Le recours à la méthode DC apparemment répandu parmi les professionnels de la santé à Kinshasa est contraire aux pratiques de référence et il se peut qu'il contribue aux complications de l'avortement qu'expérimentent les femmes.

La situation socioéconomique de la femme déterminerait, de l'avis des répondants, le type de soins d'avortement qu'elle reçoit. Les informateurs ont été invités à estimer les proportions de femmes pauvres et non pauvres qui subissent chaque type d'avortement et s'adressent à chaque type de prestataire. La moyenne de leurs réponses a ensuite été calculée pour obtenir une estimation globale. Ainsi, près de trois quarts $(71 \%)$ des femmes non pauvres qui interrompent une grossesse obtiennent une intervention chirurgicale (DC ou aspiration), 18\% utilisent le misoprostol et $11 \%$ recourent aux autres méthodes, y compris celles offertes par les prestataires non qualifiés (ingestion d'herbes, utilisation de médicaments autres que le misoprostol ou insertion d'objets dans le vagin; Figure 2.1$)^{42}$. En revanche, $24 \%$ des
FIGURE

D'après les informateurs clés, les femmes non pauvres de Kinshasa obtiennent généralement un avortement chirurgical.

Méthode d'avortement utilisée

Chirurgical* Misoprostol

Autre†
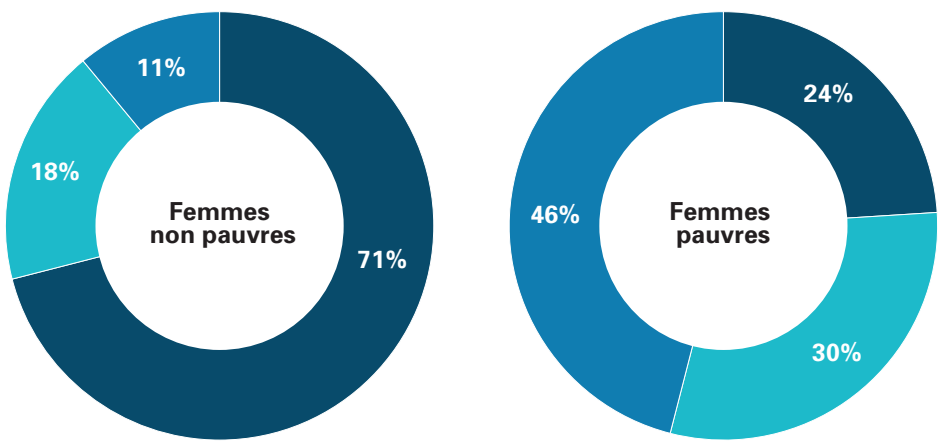

femmes pauvres obtiendraient une opération chirurgicale, $30 \%$ utiliseraient le misoprostol et $46 \%$ recourraient aux autres méthodes.

Selon l'estimation des informateurs clés, près de trois quarts des femmes pauvres qui obtiennent une intervention chirurgicale consultent des prestataires de santé qualifiés autres que des médecins, comme par exemple des infirmières ou des sages-femmes, et $27 \%$ s'adressent à un médecin ou à un clinicien (Tableau 2.1, page 12) ${ }^{42}$. En ce qui concerne les femmes non pauvres, ces proportions sont presque inverses: $78 \%$ obtiendraient leur avortement chirurgical d'un médecin, tandis que les autres s'adresseraient à d'autres prestataires qualifiés. Pour les avortements par misoprostol, il semblerait que deux tiers des femmes pauvres consultent des prestataires médicaux qualifiés mais non médecins ou des pharmaciens, tandis qu'environ la moitié des femmes non pauvres s'adressent aux médecins ou aux cliniciens. Quelque $21 \%$ des femmes pauvres et $12 \%$ de celles non pauvres obtiennent le misoprostol et se l'administrent elles-mêmes.

De l'avis des informateurs clés, les médecins et les cliniciens sont généralement les prestataires les plus onéreux de l'avortement, l'option la moins coûteuse étant celle de l'interruption de la grossesse par la femme elle-même (Figure 2.2, page $12)^{42}$. Cela dit, le montant payé par les femmes pour un avortement varie suivant leur situation de pauvreté. Pour un avortement obtenu d'un médecin ou d'un clinicien, les femmes pauvres semblent payer 74 dollars ( $d$ 'après la moyenne
NOTES FIGURE 2.1

*Dilatation et curetage, aspiration manuelle intrautérine ou aspiration électrique intra-utérine. IIngestion d'herbes ou de médicaments autres que le misoprostol, méthodes physiques ou autres moyens. Source: référence 42 
Distribution en pourcentage estimée, d'après les informateurs clés, des femmes pauvres et non pauvres qui se sont fait avorter, par type de prestataire, suivant que les femmes ont obtenu un avortement chirurgical, médical ou d'autre type, Kinshasa, 2016

\begin{tabular}{|c|c|c|c|c|c|c|}
\hline \multirow[b]{2}{*}{ TYPE DE PRESTATAIRE } & \multicolumn{2}{|c|}{ Avortement chirurgical } & \multicolumn{2}{|c|}{$\begin{array}{l}\text { Avortement par } \\
\text { misoprostol }\end{array}$} & \multicolumn{2}{|c|}{$\begin{array}{l}\text { Autres types } \\
\text { d'avortement* }\end{array}$} \\
\hline & $\begin{array}{l}\text { Femmes } \\
\text { pauvres }\end{array}$ & $\begin{array}{c}\text { Femmes } \\
\text { non } \\
\text { pauvres }\end{array}$ & $\begin{array}{l}\text { Femmes } \\
\text { pauvres }\end{array}$ & $\begin{array}{c}\begin{array}{c}\text { Femmes } \\
\text { non } \\
\text { pauvres }\end{array} \\
\text {. }\end{array}$ & $\begin{array}{l}\text { Femmes } \\
\text { pauvres }\end{array}$ & $\begin{array}{c}\begin{array}{c}\text { Femmes } \\
\text { non } \\
\text { pauvres }\end{array} \\
\text {. }\end{array}$ \\
\hline Médecin ou clinicien & 27 & 78 & 15 & 47 & 1 & 7 \\
\hline $\begin{array}{l}\text { Personnel infirmier, sage-femme } \\
\text { ou autre prestataire qualifié non } \\
\text { médecin }\end{array}$ & 71 & 21 & 39 & 27 & 11 & 14 \\
\hline $\begin{array}{l}\text { Praticien traditionnel ou autre } \\
\text { prestataire non qualifié }\end{array}$ & 2 & 0 & 2 & 0 & 34 & 16 \\
\hline Pharmacien & 1 & 0 & 24 & 13 & 11 & 17 \\
\hline Avortement auto-provoqué & 0 & 0 & 21 & 12 & 42 & 45 \\
\hline TOTAL & 100 & 100 & 100 & $99+$ & 100 & 100 \\
\hline
\end{tabular}

NOTES *Ingestion d'herbes ou de médicaments autres que le misoprostol, méthodes physiques ou autres moyens. tLa distribution n'atteint pas $100 \%$ pour cause d'erreur d'arrondissement et de faible maîtrise du calcul de la part du répondant ou du collecteur de données lors de la collecte. N.B.: Les données proviennent d'entretiens avec 113 informateurs clés. Source: référence 42.

des réponses des informateurs) ${ }^{*}$, alors que celles non pauvres payeraient environ trois fois plus (223 dollars). La différence du coût estimé d'un groupe socioéconomique à l'autre se réduit au fur et à mesure que le niveau de qualification du prestataire diminue. II semblerait que les femmes non pauvres paient environ deux fois plus que celles qui sont pauvres pour un avortement obtenu d'un prestataire non médecin (79 vs 35 dollars) et un peu moins du double pour une intervention obtenue d'un praticien traditionnel ou autre prestataire non qualifié (30 vs 18 dollars). L'avortement pratiqué par la femme elle-même est estimé coûter environ cinq dollars pour les femmes non pauvres et trois dollars pour celles pauvres.

\section{Déclarations par les femmes de leur expérience de l'avortement}

L'Enquête prospective de morbidité menée dans le cadre de l'Étude de 2016 sur l'avortement à Kinshasa a invité les femmes en soins après avortement dans les formations sanitaires à indiquer si leur grossesse avait été interrompue du fait d'un acte qu'elles ou que quelqu'un d'autre avaient posé. Environ deux cinquièmes des participantes ont répondu affirmativement à cette question ${ }^{44}$. II est cependant connu que les femmes sous-déclarent souvent l'avortement en raison de sa stigmatisation $^{45-47}$. Aussi les chercheurs de l'Étude de 2016 sur l'avortement à Kinshasa ont-ils appliqué un système de classification initialement mis au point

\section{FIGURE}

D'après les informateurs clés, le coût d'un avortement varie selon le type de prestataire et le degré de pauvreté des femmes.

Coût estimé (en dollars)

* Toutes les sommes monétaires de l'Étude de 2016 sur l'avortement à Kinshasa sont libellées en dollars américains de 2016 .
223

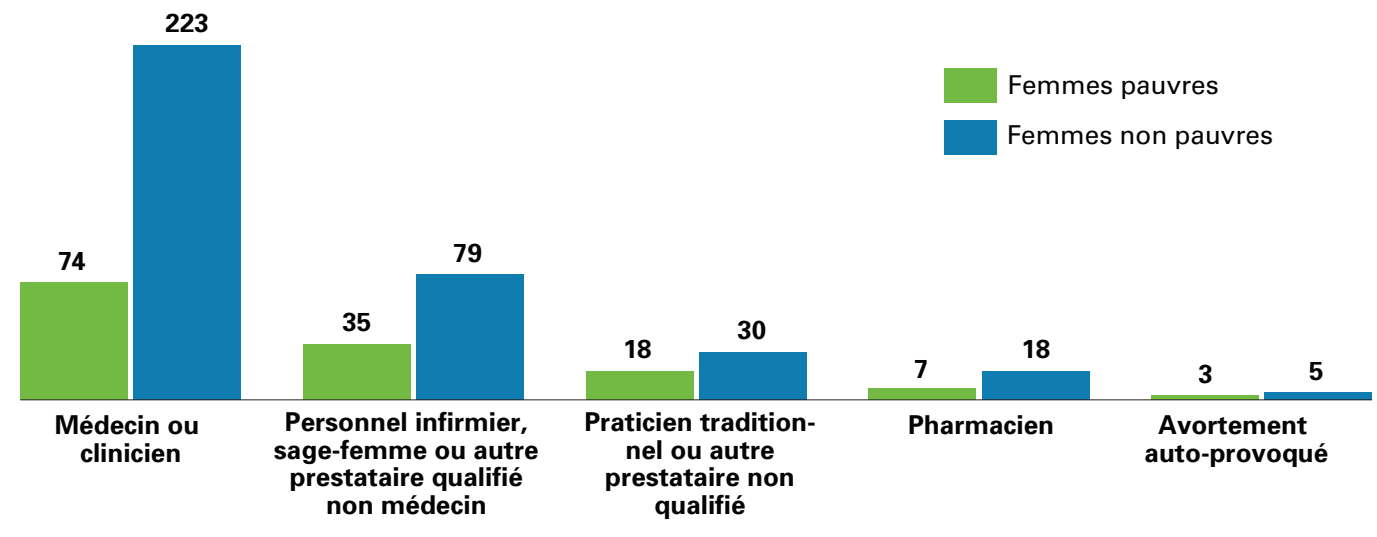

NOTE FIGURE 2.2 Les sommes monétaires sont libellées en dollars américains de 2016. Source: référence 42. 
par l'Organisation mondiale de la Santé pour incorporer tant l'auto-déclaration des femmes que les observations des agents de santé (Tableau 1 en annexe disponible en ligne) ${ }^{48}$. Ce système leur a permis d'estimer que $72 \%$ des patientes en soins après avortement interviewées en 2016 à Kinshasa s'étaient fait avorter ${ }^{6}$.

Parmi les femmes classifiées ainsi, $81 \%$ ont indiqué n'avoir pas planifié leur grossesse au moment de la conception ${ }^{44}$. Ces 452 femmes (ayant déclaré n'avoir pas désiré, avoir désiré plus tard ou ne pas savoir si elles désiraient la grossesse pour laquelle elles se trouvaient alors en soins après avortement) ont invoqué plusieurs raisons de vouloir éviter une grossesse, notamment leur célibat (42\%), le désir de préserver l'honneur de leur famille (23\%), des considérations d'ordre financier (22\%), la peur d'être mises à la porte (21\%), des préoccupations de santé (19\%), le désir d'espacer les naissances (19\%), leur trop jeune âge pour être mère (15\%), le fait d'avoir déjà assez d'enfants (13\%), l'opposition de la famille (11\%) ou l'opposition du partenaire (11\%; Figure 2.3). La plupart de ces raisons sont aussi celles invoquées par le petit groupe de femmes qui avaient dit avoir initialement désiré la grossesse mais avoir ensuite changé d'avis.

Près de la moitié (44\%) des femmes classifiées comme ayant subi un avortement ont déclaré avoir discuté de l'interruption de leur grossesse à un certain moment avant l'obtention des soins dans la formation où elles se trouvaient (données non indiquées) $)^{44}$. Le plus souvent, les femmes en avaient parlé avec leur partenaire (73\%), avec une amie (33\%) ou avec une parente (31\%). Selon la majorité de ces femmes (83\%), au moins une personne consultée pensait qu'elles devraient interrompre la grossesse.

Trois cinquièmes des femmes classifiées comme ayant subi un avortement ont déclaré explicitement avoir agi pour interrompre leur grossesse. Deux cinquièmes d'entre elles ont dit en avoir pris la décision seules, tandis que les autres avaient le plus souvent consulté leur partenaire ${ }^{44}$. Environ deux cinquièmes des femmes qui avaient dit avoir agi pour interrompre leur grossesse avaient eu recours à plus d'une méthode, en déclarant parfois jusqu'à cinq (Figure 2.4, page 14). Plus de la moitié (56\%) ont déclaré avoir utilisé le misoprostol, comme seule méthode (31\%; données non indiquées) ou parmi d'autres (25\%).
FIGURE

\section{Les femmes qui se sont fait avorter ont déclaré différentes raisons de rejet de la grossesse au moment où elle est survenue.}

$\%$ mentionnant chaque raison

Femme célibataire

Désir de préserver l'honneur de la famille

Considérations financières

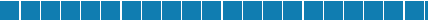

Peur d'être exclue du foyer

Raisons de santé

पा

Désir d'espacement des naissances

\section{Thir d'espacement des na}

La femme se sent trop jeune pour avoir un enfant

La femme a assez d'enfants

(1)

Les membres de la famille s'opposent à la grossesse

Le partenaire rejette la grossesse

पा

Le père est un partenaire de passage

(I)

Peur d'être exclue de l'école

Femme abandonnée par son partenaire

(1)

Désir de continuer à travailler

NOTES FIGURE 2.3 Le dénominateur représente les 452 femmes de l'Enquête prospective de morbidité de 2016 classifiées comme ayant subi un avortement et ayant déclaré qu'elles ne désiraient pas, désiraient plus tard ou ne savaient pas si elles désiraient la grossesse à l'origine des soins obtenus. Les pourcentages calculés avec pondération. Source: référence 44. 
Parmi des patientes en soins après avortement ayant déclaré avoir agi pour interrompre leur grossesse, plus de la moitié ont utilisé le misoprostol et les deux cinquièmes ont eu recours à plus d'une méthode.

\section{Méthodes utilisées pour provoquer l'avortement*}

Misoprostol

Prise orale d'herbes ou d'autres remèdes maison

Autres comprimés (non spécifiés)

Injection

Dilatation et curetage

Oxytocine

Insertion vaginale d'herbes, de substances ou d'autres matières

Aspiration manuelle ou électrique intra-utérine

Autres méthodes

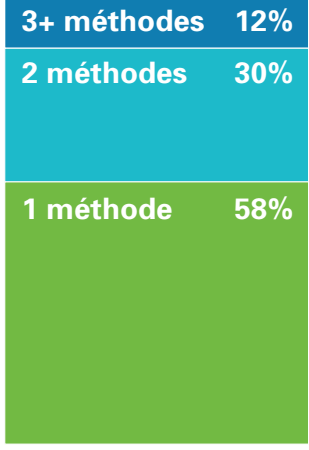

Nombre de méthodes utilisées pour provoquer l'avortement

NOTES FIGURE 2.4 *Plus d'une réponse était permise. †Méthodes telles que lavement à base de plantes, activité physique excessive ou massage vigoureux. N.B.: Les pourcentages sont calculés avec pondération; le dénominateur est 327. Source: référence 44.

Il importe de noter que les données de l'Enquête prospective de morbidité représentent le sous-ensemble des femmes atteintes de complications d'un avortement non médicalisé qui avaient réussi à obtenir des soins dans une formation sanitaire. Bien qu'il nous semble peu probable que les tendances et raisons décisionnelles d'interruption de la grossesse des femmes de ce groupe aient différé de celles des femmes ayant avorté sans complications ou subissant des complications non traitées, les méthodes abortives utilisées par ces groupes étaient vraisemblablement différentes. Par exemple, les femmes n'ayant pas connu de complications ont peut-être eu recours à des méthodes moins risquées que celles captées dans cette étude. 


\section{(3) Conséquences sanitaires de l'avortement non médicalisé et soins après avortement}

Selon les estimations, 6,2 millions d'avortements ont eu lieu chaque année en Afrique au cours de la période $2010-2014^{49}$ et $69 \%$ de ceux pratiqués en Afrique centrale - la sous-région dont fait partie Kinshasa - se classent dans la catégorie « la moins sûre » (par rapport aux interventions « sûres » ou « moins sûres » ${ }^{\dagger}$ ). En d'autres termes, les avortements pratiqués en Afrique centrale le sont, pour la majorité, par des prestataires non qualifiés et selon des méthodes non médicalisées. Dix-neuf pour cent des avortements pratiqués en Afrique centrale sont « moins sûrs », ce qui veut dire qu'ils le sont par des prestataires non qualifiés ou selon des méthodes non médicalisées. Douze pour cent seulement des avortements pratiqués dans la région sont considérés sûrs.

Étant donné son haut niveau d'avortements non médicalisés, l'Afrique est le continent qui présente le plus haut taux de létalité de l'intervention, à 141 décès pour 100000 avortements ${ }^{50}$. L'Afrique enregistre $29 \%$ de la totalité des avortements non médicalisés, mais $62 \%$ des décès imputables à ces avortements à l'échelle mondiale ${ }^{49}$. Selon les estimations, $7 \%$ des décès maternels survenus en Afrique subsaharienne en 2017 étaient liés à l'avortement ${ }^{51}$, soit une perte largement évitable de quelque 14600 vies, dont 3000 en Afrique centrale. Les études réalisées dans les contextes africains ont montré que, au niveau du ménage, le décès maternel — du fait d'un avortement non médicalisé ou d'autres causes - peut mener à l'insécurité économique, à une santé infantile précaire et à l'amoindrissement du revenu, de l'éducation et des occasions d'épanouissement ${ }^{52-56}$. Ces conséquences négatives de l'avortement non médicalisé se répercutent sur le bien-être des communautés et des pays ${ }^{57,58}$.

Bien que la mort en soit la conséquence la plus grave, l'avortement non médicalisé peut conduire à toute une série de complications, exigeant pour certaines une attention médicale afin d'éviter douleurs et incapacité à court ou à long terme et qualité de vie réduite $\mathrm{e}^{59,60}$. Les soins après avortement forment un paquet de services de santé vitaux ciblant les besoins particuliers des femmes atteintes de complications d'un avortement ou d'une fausse couche. Pour 2014, les estimations indiquent que la satisfaction totale du besoin de soins après avortement dans les régions en développement aurait fortement réduit le nombre de décès imputables à l'avortement non médicalisé, de 22000 à 9000 par an ${ }^{61}$. De plus, la pleine prestation de soins après avortement aurait largement amélioré le bien-être des femmes en réduisant le nombre d'années de vie saine perdues pour cause d'avortement $^{\ddagger}$ de 1,5 million à 600000 par an.

\section{Soins après avortement à Kinshasa}

L'Étude de 2016 sur l'avortement à Kinshasa jette la lumière sur la nécessité de soins après avortement et sur l'accès à ces soins dans la ville ${ }^{5}$. Après correction des patientes susceptibles d'avoir été comptées deux fois du fait de la référence d'un établissement des soins vers un autre et des femmes identifiées comme extérieures à la ville, il a été estimé que 49090 Kinoises avaient reçu des soins après avortement par suite d'un avortement ou d'une fausse couche. Parmi ces femmes, 37870 ont été traitées pour cause de complications résultant spécifiquement d'un avortement, indiquant que $77 \%$ de la totalité des femmes ayant reçu des soins après avortement à Kinshasa en 2016 avaient requis ces soins pour cause de complications liées à l'avortement non médicalisé. Le taux de traitement en soins après avortement était de 14 femmes traitées pour 1000 femmes âgées de 15 à 49 ans.

Les informateurs clés du Sondage des professionnels de la santé ont estimé la proportion de femmes qui auraient eu besoin de soins après avortement mais n'en ont pas obtenus. D'après leur connaissance des méthodes utilisées pour provoquer l'avortement, de la sécurité des méthodes
† Une nouvelle définition de la sécurité de l'avortement enoncee en 2017 classe les procédures pratiquées dans trois catégories: la moins sûre, moins sure et sûre. Ce changement de définition étant intervenu après la réalisation de I'Étude de 2016 sur l'avortement à Kinshasa, nos données font référence à l'avortement " non médicalisé ", une désignation équivalant aux catégories u moins sûre " et " moins sûre " combinées.

‡ Mesuré en années de vie corrigées de l'incapacité (AVCI), une mesure conçue pour faciliter la comparaison des charges de décès prématurés et d'incapacité attribuables à différentes causes dans les contextes considérés. Les AVC imputables aux complications de l'avortement peuvent largement être évitées. 
3.1 Selon les estimations, une plus grande proportion de femmes pauvres, par rapport à celles non pauvres, souffrent de complications liées à l'avortement mais une plus faible proportion de ces femmes obtiennent les soins après avortement requis.

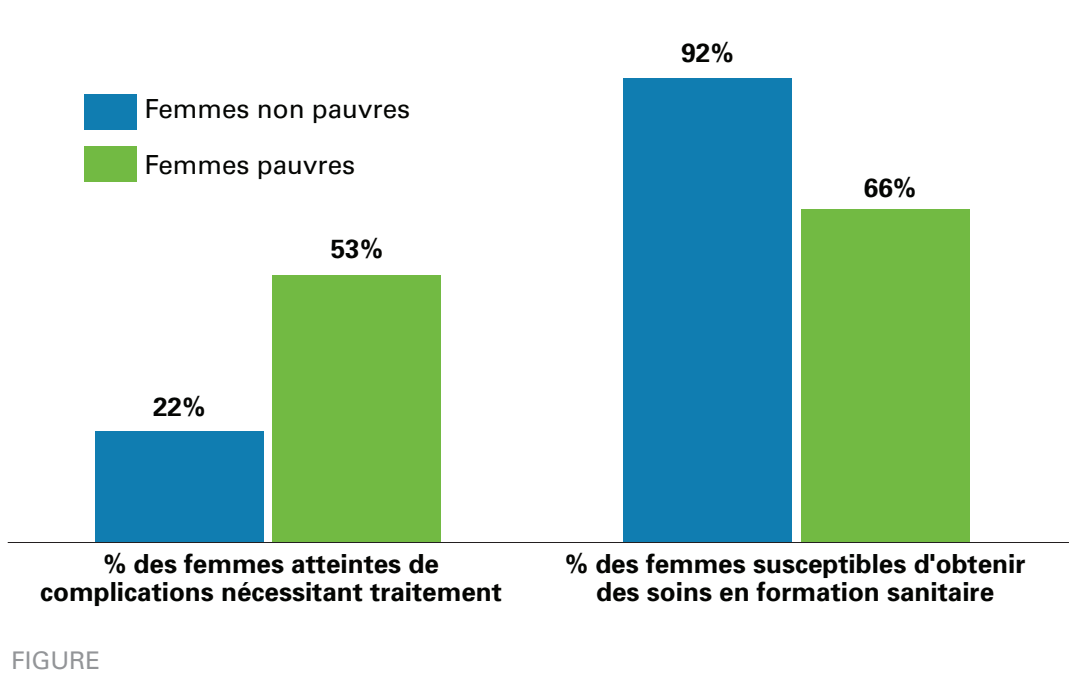

3.2

Pratiquement tous les soins après avortement assurés à Kinshasa en 2016 l'ont été dans des formations sanitaires privées.

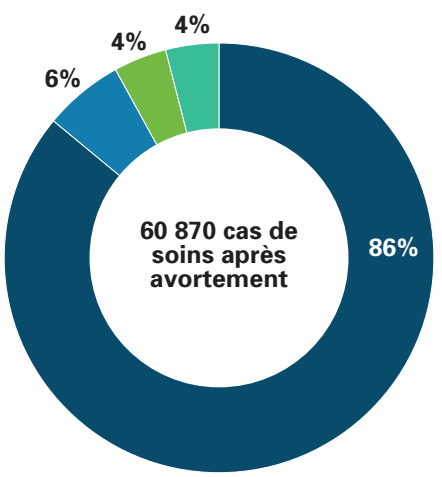

Formation sanitaire assurant des soins après avortement

Centre de santé privé/d'ONG

Hôpital privé/d'ONG

Hôpital public

Centre de santé public

NOTE FIGURE 3.1

Les pourcentages reposent sur l'opinion experte des informateurs clés du Sondage des professionnels de la santé. Source: référence 42 .

NOTE FIGURE 3.2 Les pourcentages sont calculés avec pondération. Source: référence 5 . et de la probabilité que les femmes atteintes de complications obtiennent des soins après avortement, ils ont estimé que $34 \%$ des femmes qui interrompent une grossesse ont vraisemblablement des complications suffisamment graves pour justifier un traitement en formation sanitaire ${ }^{42,62}$. Parmi ce groupe de femmes, estiment-ils, $77 \%$ obtiennent ce traitement. Les $23 \%$ restants (environ 11500 femmes) obtiennent soit des soins en dehors du système de soins formel (auprès de professionnels médicaux ou de praticiens traditionnels) ou elles n'en obtiennent aucun.

Les informateurs clés du Sondage des professionnels de la santé laissent aussi entendre que le besoin de soins après avortement et le recours à ces soins varient suivant les caractéristiques socioéconomiques et reflètent de profondes inégalités d'accès aux soins et de résultat. Selon leurs estimations, environ la moitié des femmes pauvres qui se font avorter souffrent de complications nécessitant un traitement, par rapport à $22 \%$ de leurs homologues non pauvres (Figure 3.1 ${ }^{42}$ Parmi celles atteintes de complications, près de $66 \%$ des femmes pauvres obtiendraient les soins requis en formation sanitaire, par rapport à $92 \%$ de celles non pauvres. Ainsi, un pourcentage estimé à $18 \%$ des femmes pauvres souffrent de complications non traitées en formation sanitaire, par rapport à $2 \%$ seulement des femmes non pauvres.

Les données de l'Enquête dans les formations sanitaires montrent que presque tous les soins après avortement à Kinshasa (92\%) sont assurés dans les formations du secteur privé (ONG incluses; Figure 3.2) et que les coûts à la charge de la patiente y sont probablement plus élevés que dans les formations du secteur public ${ }^{5}$. Bien que la formation sanitaire ayant atteint le plus grand nombre annuel de cas de soins après avortement (252 femmes) eût été un hôpital de l'État, dans l'ensemble, un peu moins de $8 \%$ des cas avaient été traités dans les hôpitaux et les centres de santé du secteur public.

\section{Caractéristiques des patientes recevant des soins après avortement}

La recherche n'a guère décrit, dans le passé, les caractéristiques des femmes qui obtiennent des soins après avortement, en RDC en général ou à Kinshasa en particulier. L'une des études réalisées a examiné une cohorte de femmes traitées pour complications d'avortements clandestins dans les cliniques universitaires de Kinshasa en 1978$1979^{63}$. Parmi les femmes qui avaient interrompu illégalement leur grossesse, deux groupes étaient disproportionnément représentés: les femmes de moins de 20 ans enceintes pour la première ou la deuxième fois, célibataires, étudiantes ou pauvres, et celles âgées de 20 ans ou plus mariées, instruites et économiquement actives. Toutes les patientes ont été considérées avoir obtenu l'intervention avant la $12^{\mathrm{e}}$ semaine de gestation, généralement auprès de prestataires non qualifiés. Une seconde étude, menée à Kinshasa en 2014, s'est concentrée sur les femmes admises aux services d'obstétrique d'urgence de cinq grands hôpitaux de référence ${ }^{64}$. Parmi ces femmes, près de $15 \%$ souffraient de complications d'un avortement. La majorité des patientes étaient mariées ou vivaient en couple, 
avaient déjà plus d'un enfant et n'ont déclaré aucun avortement antérieur. Par ailleurs, les adolescentes et les femmes célibataires étaient plus susceptibles d'avoir été admises pour cause de complications d'un avortement que les patientes plus âgées ou que les femmes mariées, respectivement.

L'Enquête prospective de morbidité de l'Étude de 2016 sur l'avortement à Kinshasa éclaire davantage les caractéristiques des femmes qui reçoivent des services après avortement, grâce aux données tirées d'entretiens avec les femmes qui avaient obtenu des soins après un avortement ou une fausse couche. Au moment de l'interview, la moitié des femmes concernées avaient entre 20 et 29 ans, 35\% avaient 30 ans ou plus et $16 \%$ étaient des adolescentes âgées de 15 à 19 ans (Tableau 3.1, page 18$)^{6}$. Plus de la moitié étaient en union (58\%) et une grande majorité (87\%) avaient atteint pour le moins un certain niveau d'éducation secondaire. Quatre sur 10 étaient pauvres. Plus du quart (28\%) des femmes obtenant des soins après avortement ont déclaré pratiquer la contraception au moment de la conception et une grande majorité avoir été au premier trimestre de la grossesse (81\%) au moment de l'avortement ou de la fausse couche. La majorité des femmes en soins après avortement ont aussi déclaré avoir eu au moins une grossesse antérieure (81\%), indiquant par ailleurs qu'elles avaient au moins un enfant $(69 \%$; données non indiquées $)^{6,44}$. Environ un quart (27\%) ont déclaré au moins un avortement antérieur ${ }^{6}$.

Le sous-ensemble des patientes ayant explicitement déclaré s'être fait avorter était composé de femmes généralement plus jeunes que les patientes en soins après avortement dans leur ensemble ${ }^{44}$. Près de trois cinquièmes (59\%) des femmes ayant déclaré avoir interrompu leur grossesse étaient âgées de 20 à 29 ans, tandis que les adolescentes et les femmes de 30 ans et plus représentaient, respectivement, un cinquième pour chaque groupe. D'importantes proportions étaient célibataires (58\%), pratiquaient la contraception au moment de la conception (36\%), en étaient au premier trimestre de la grossesse (90\%) et s'étaient déjà fait avorter au moins une fois (39\%).

\section{Gravité des complications traitées}

L'Enquête prospective de morbidité a classé les patientes en soins après avortement en fonction de la gravité de leurs complications (voir les critères de classification au Tableau 2 en annexe $)^{6}$. Cinq pour cent ne présentaient aucun signe distinct de

\subsection{Près de deux tiers des femmes en soins après avortement dans les formations sanitaires de Kinshasa en 2016 souffraient de complications graves ou de gravité moyenne.}

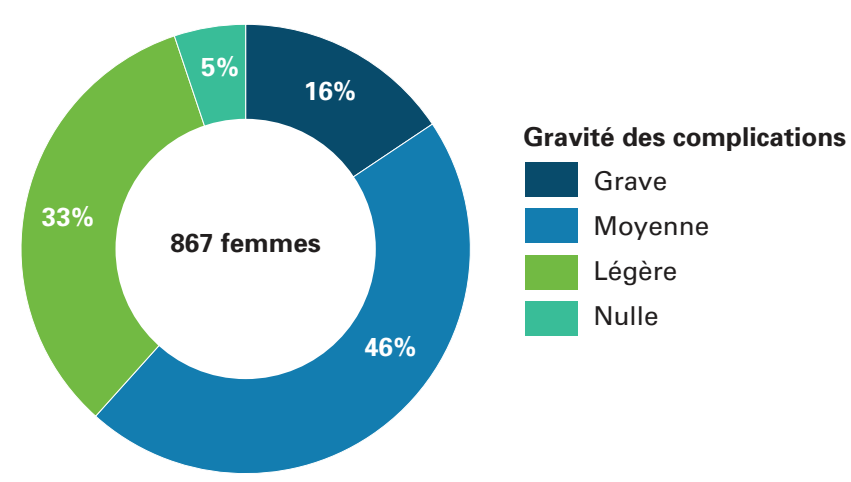

complications et avaient probablement cherché inutilement à obtenir une assistance médicale, $33 \%$ souffraient de légères complications, $46 \%$ de complications de gravité moyenne et les $16 \%$ restants de complications graves telles qu'un état de choc, la défaillance d'organe, la péritonite généralisée ou la mort (Figure 3.3).

La gravité des complications variait suivant les caractéristiques socioéconomiques, démographiques et cliniques des femmes ${ }^{6}$. La probabilité d'avoir présenté une complication de gravité moyenne ou sévère était de $49 \%$ moins élevée parmi les femmes âgées de 20 à 24 ans, par rapport à celles de 35 ans et plus. De plus, la probabilité d'avoir présenté des complications graves était de $71 \%$ moins élevée parmi les femmes âgées de 25 à 29 ans que chez les femmes de 35 ans et plus. Les femmes pauvres et celles ayant obtenu un avortement présentaient une probabilité double de connaître des complications moyennes ou graves, par rapport aux femmes mieux loties et à celles ayant fait une fausse couche, respectivement. Les femmes célibataires ou en union avait une probabilité deux fois plus élevée de présenter des complications moyennes ou graves que leurs homologues mariées ou précédemment mariées. Pour celles ayant déclaré avoir dépassé le premier trimestre de la grossesse au moment de l'interruption, la probabilité de complications graves était 3,7 fois supérieure à celle des femmes avortées au premier trimestre. Enfin, la probabilité de présenter une complication grave était deux fois moins élevée parmi les femmes ayant déjà avorté comparativement à celles n'ayant jamais avorté.

\section{NOTE FIGURE 3.3}

Les pourcentages sont calculés avec pondération Source: référence 6 
1 Caractéristiques sélectionnées de femmes recevant des soins après avortement dans les formations sanitaires, Kinshasa, 2016

\begin{tabular}{|c|c|c|c|c|}
\hline \multirow[b]{2}{*}{ CARACTÉRISTIQUE } & \multicolumn{2}{|c|}{$\begin{array}{l}\text { Toutes les femmes recevant des } \\
\text { soins après avortement }\end{array}$} & \multicolumn{2}{|c|}{$\begin{array}{l}\text { Femmes en soins après avortement } \\
\text { ayant déclaré avoir agi pour } \\
\text { interrompre leur grossesse }\end{array}$} \\
\hline & Nombre & Distribution en $\%$ & Nombre & Distribution en $\%$ \\
\hline \multicolumn{5}{|l|}{ Âge } \\
\hline 15 à 19 ans & 112 & 16 & 61 & 20 \\
\hline 20 à 24 ans & 191 & 23 & 108 & 35 \\
\hline 25 à 29 ans & 202 & 26 & 71 & 24 \\
\hline 30 à 34 ans & 158 & 17 & 38 & 11 \\
\hline 35 à 49 ans & 177 & 18 & 43 & 11 \\
\hline \multicolumn{5}{|l|}{ Situation matrimoniale } \\
\hline Célibataire & 299 & 38 & 194 & 58 \\
\hline En union & 534 & 58 & 115 & 35 \\
\hline Antérieurement mariée & 34 & 4 & 20 & 7 \\
\hline \multicolumn{5}{|l|}{ Résidence } \\
\hline Kinshasa & 838 & 97 & 318 & 97 \\
\hline Hors Kinshasa & 29 & 3 & 11 & 3 \\
\hline \multicolumn{5}{|l|}{ Éducation } \\
\hline Primaire ou moins & 110 & 13 & 37 & 11 \\
\hline Secondaire incomplète & 308 & 36 & 134 & 42 \\
\hline Secondaire accomplie & 288 & 35 & 100 & 33 \\
\hline Supérieure & 161 & 16 & 58 & 15 \\
\hline \multicolumn{5}{|c|}{ Situation de pauvreté du ménage } \\
\hline Pauvre & 316 & 40 & 122 & 38 \\
\hline Non pauvre & 551 & 60 & 207 & 62 \\
\hline \multicolumn{5}{|l|}{ Avortements antérieurs } \\
\hline 0 & 637 & 73 & 203 & 61 \\
\hline$\geq 1$ & 229 & 27 & 126 & 39 \\
\hline \multicolumn{5}{|c|}{$\begin{array}{l}\text { Pratique contraceptive au moment } \\
\text { de la grossesse }\end{array}$} \\
\hline Oui & 246 & 28 & 118 & 36 \\
\hline Non & 621 & 72 & 211 & 64 \\
\hline \multicolumn{5}{|l|}{ Âge gestationnel } \\
\hline Premier trimestre & 654 & 80 & 275 & 90 \\
\hline Après le premier trimestre & 189 & 20 & 42 & 10 \\
\hline TOTAL & 867 & 100 & 329 & 100 \\
\hline
\end{tabular}

NOTES Les données manquantes ayant été exclues, les nombres de femmes ne correspondent ainsi pas, pour certaines mesures, au total. La catégorie "En union " comprend les femmes mariées (environ $40 \%$ ) et les femmes en concubinage (18\%). Les pourcentages sont calculés avec pondération. Source: référence 6 . 
Nouvelle perspective sur les services de soins après avortement

L'Enquête prospective de morbidité a également recueilli les données en 2016 sur le type de services obtenus par les femmes en soins après avortement. Environ la moitié (54\%) des femmes en quête de soins après avortement avaient été traitées par des médecins, prestataires les plus probables des femmes atteintes de complications graves ${ }^{6}$. Bien que non recommandée par l'Organisation mondiale de la Santé, la dilatation et curetage (DC) s'est avérée la technique le plus souvent pratiquée sur les femmes recevant des soins après avortement (49\% des cas; Figure 3.4). Le curetage digital avait été utilisé pour $23 \%$ des patientes, I'aspiration manuelle ou électrique pour $14 \%$ et le misoprostol et autres méthodes pour les $14 \%$ de cas restants. La DC était plus courante pour les interventions pratiquées durant le premier trimestre (51\%) que pour celles des deuxième et troisième trimestres (43\%; données non indiquées) ${ }^{44}$.

Onze pour cent seulement des femmes en soins après avortement avaient reçu un médicament analgésique. Cette médication était légèrement plus courante parmi les femmes souffrant de complications graves (15\%) que parmi celles sans complications ( $9 \%$ ) ou présentant une complication légère $(11 \%)^{6}$. La non administration d'analgésiques a aussi été documentée dans les contextes de prestation de services d'avortement médicalisés et les données semblent indiquer une pratique attribuable peut-être au biais à l'encontre des femmes qui ont interrompu une grossesse ${ }^{65}$.

Enfin, 15\% seulement des patientes comprises dans l'Enquête prospective de morbidité de l'Étude de 2016 sur l'avortement à Kinshasa avaient reçu une méthode contraceptive avant de quitter la formation sanitaire, tandis que $16 \%$ étaient orientées vers une autre source. La fourniture de contraceptifs était plus courante parmi les femmes atteintes de complications graves (20\%) que parmi celles sans complications ou atteintes de légères complications $(13 \%)^{6}$.
3.4 Parmi les femmes recevant des soins après avortement à Kinshasa, la méthode de dilatation et curetage (DC) est la forme de traitement la plus courante.

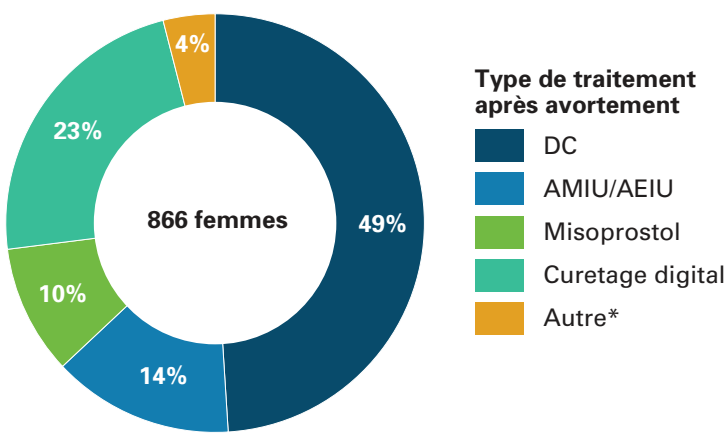

NOTES FIGURE 3.4 *Évacuation forceps ou par autres moyens. N.B.: Les pourcentages sont calculés par pondération. $\mathrm{DC}=$ Dilatation et curetage. $\mathrm{AMIU}=\mathrm{Aspiration}$ manuelle intra-utérine. $\mathrm{AEIU}=\mathrm{Aspiration}$ électrique intra-utérine. Source: référence 6. 


\section{Facteurs contribuant}

\section{à l'avortement non}

médicalisé

Pour déterminer comment réduire la morbidité et la mortalité imputables à l'avortement non médicalisé, il faut d'abord en comprendre les causes. Globalement, le taux d'avortement médicalisé ou non dans un contexte donné est influencé par plusieurs facteurs: préférences de fécondité des femmes, tendances de la pratique contraceptive ou non, motivation des femmes à éviter de mener à terme une grossesse non planifiée et capacité d'agir sur leurs préférences compte tenu du contexte légal, structurel et social ${ }^{66}$.

\section{Pratique contraceptive et obstacles}

Les données de l'Enquête démographique et de santé (EDS) 2013-2014 révèlent une pratique contraceptive moderne faible en RDC et un besoin non satisfait de contraception élevé 9 . Seuls $8 \%$ des femmes mariées et $21 \%$ de celles célibataires sexuellement actives dans le pays pratiquaient une méthode de contraception moderne au moment de l'enquête et les méthodes modernes étaient pratiquées par 19\% des femmes mariées à Kinshasa. Vingt-trois pour cent des Kinoises mariées présentaient un besoin non satisfait de contraception; la plupart dans le but d'espacer leurs maternités plutôt que de les éviter ou de les arrêter. Une proportion importante des Kinoises mariées (26\%) recouraient, de plus, aux méthodes de planification familiale traditionnelles. La pratique des méthodes modernes répondait à $28 \%$ seulement du besoin contraceptif des femmes mariées. Le besoin non satisfait parmi les Kinoises célibataires sexuellement actives était de $31 \%$ et les méthodes modernes répondaient à $30 \%$ du besoin contraceptif de ce groupe.

Les caractéristiques de niveau individuel et du ménage contribuent largement à l'adoption de la contraception. Selon les données nationales de l'EDS 2013-2014, la prévalence contraceptive (pratique des méthodes modernes et traditionnelles) était supérieure parmi les femmes davantage instruites et parmi celles des ménages plus riches, par rapport aux femmes moins instruites et plus pauvres 9 . Dans une étude menée à Kinshasa en 2006 , le fait d'avoir déjà pratiqué une méthode moderne et d'avoir parlé de la contraception avec autrui au cours des 12 mois précédents était associé à un niveau d'adoption supérieur ${ }^{24}$. La pratique variait aussi en fonction de l'âge. Ainsi, les adolescentes âgées de 15 à 19 ans étaient moins informées sur les méthodes modernes et par conséquent moins susceptibles de les pratiquer que leurs aînées. Curieusement, selon les données d'une étude nationalement représentative de la RDC en 2015, l'exposition à la violence sexuelle causée par un partenaire intime était associée positivement à la pratique d'une méthode moderne, alors que l'exposition à d'autres formes de conflit ne l'était pas (en raison peut-être du faible accès dans les zones en proie au conflit) ${ }^{67}$.

Les facteurs de structure et l'environnement jouent aussi un rôle dans la pratique contraceptive ou non des femmes et des couples. Lors d'entretiens qualitatifs organisés dans six provinces de la RDC, les hommes et les femmes ont signalé le manque d'accès à l'information comme obstacle à la pratique contraceptive ${ }^{68}$. Les répondants ont spécifiquement mentionné la peur des effets secondaires, le manque de connaissance sur les types et les sources de méthodes contraceptives et la désinformation - tous reflets de l'information insuffisante fournie dans les formations sanitaires et ailleurs. Le coût d'une consultation ou de l'approvisionnement associé à une visite contraceptive peut aussi entraver l'accès. Lors de l'EDS 2013-2014, 57\% des femmes qui pratiquaient une méthode moderne ont déclaré l'obtenir auprès d'une structure privée, généralement une pharmacie, laissant entendre que malgré l'existence de services de contraception gratuits ou subventionnés en RDC, la plupart des femmes n'accèdent pas à ce type de services ${ }^{9}$.

De plus, beaucoup de femmes peuvent trouver que les méthodes ou services dont elles ont besoin ne sont pas disponibles dans les structures, fussent-elles publiques ou privées. Lors des 
enquêtes de suivi de performance et de redevabilité PMA 2020 des années 2014, 2015 et 2016, un cinquième des formations sanitaires publiques et privées et des pharmacies de Kinshasa qui déclaraient offrir des services de planification familiale ne les offraient de fait pas le jour de l'enquête ${ }^{69}$. L'enquête de 2016 indique que la moitié seulement des structures offrant la planification familiale avaient au moins trois méthodes disponibles et plus du quart de ces structures déclaraient avoir connu des ruptures de stocks, à l'exception du préservatif, durant les trois mois précédents.

En dépit de ces difficultés, le niveau de la pratique contraceptive semble en hausse à Kinshasa. Malgré une faible amélioration de l'adoption de la contraception entre les EDS 2007 et 2013-2014' ${ }^{\circ}$ I'enquête PMA 2020 de l'année 2017 estime que 41\%, sur l'ensemble des femmes de la ville, pratiquaient une forme de contraception et que $22 \%$ recouraient aux méthodes modernes ${ }^{10}$. Parmi les femmes mariées, ces proportions étaient de $47 \%$ et $27 \%$, respectivement. Le besoin non satisfait, pour cette année, était de $16 \%$ sur l'ensemble des femmes et de $25 \%$ parmi celles mariées.

\section{Grossesse non planifiée}

Parmi les femmes qui ne désirent pas concevoir, l'absence de contraception ou son échec peuvent mener à une grossesse inopportune ou non désirée. Face à une grossesse non planifiée, beaucoup de femmes cherchent à se faire avorter ou accouchent d'un enfant qu'elles n'avaient pas prévu. Ainsi, les données de l'EDS 2013-2014 indiquent que les Kinoises avaient en moyenne 4,2 enfants mais déclaraient en désirer 3,69.

Il y aurait eu environ 563100 grossesses à Kinshasa en 2016, d'après les estimations tirées de l'Étude de 2016 sur l'avortement à Kinshasa ${ }^{5}$. Soixanteet-un pour cent de ces grossesses n'étaient pas planifiées (Tableau 4.1). Ces chiffres correspondent, respectivement, à un taux de grossesse et de grossesses non planifiées de 241 et de 147 pour 1000 femmes âgées de 15 à 49 ans.

Ce taux de grossesses non planifiées de 147 pour 1000 femmes âgées de 15 à 49 ans à Kinshasa est élevé par rapport au taux mondial (62 pour 1000 femmes âgées de 15 à 44 ans en 2010-2014) et à ceux de tous les pays en développement (65) et d'Afrique centrale $(103)^{70}$. Parmi les grandes villes d'Afrique subsaharienne, le taux de grossesses non planifiées de Kinshasa est supérieur à ceux
TABLEAU

4.1 Indicateurs sélectionnées de grossesse et grossesse non planifiée, Kinshasa, 2016

MESURE

Nombre de grossesses

Taux de grossesse (nombre pour 1000 femmes âgées de 15 à 49 ans)

Nombre de grossesses non planifiées

343085

Taux de grossesse non planifiée (nombre pour 1000 femmes âgées de 15 à 49 ans)

$\%$ de grossesses non planifiées $61 \%$

\% de grossesses non planifiées terminées par un avortement

NOTE Source: référence 5 .

des groupes de femmes de même âge ou d'âge similaire à Addis Abeba $(123)^{34}$ et à Kampala $(132)^{35}$, et inférieur à celui de Kigali $(172)^{36}$.

\section{Caractéristiques des femmes qui choisissent l'avortement}

Les caractéristiques des Kinoises qui choisissent d'interrompre une grossesse non désirée ou inopportune ne sont indiquées que dans quelques études, assez anciennes pour la plupart. D'après une étude effectuée en 1984 à Kinshasa concernant les femmes se présentant dans les hôpitaux universitaires pour un « avortement thérapeutique » ou pour cause de complications liées à un avortement ou à une fausse couche, les deux groupes de femmes les plus susceptibles d'avoir subi un avortement étaient celles de moins de 20 ans célibataires, étudiantes ou pauvres et qui n'avaient pas eu plus de deux grossesses, d'une part, et, d'autre part, celles âgées de 20 ans ou plus qui étaient mariées, instruites et économiquement actives ${ }^{63}$. En 1990, une enquête auprès des ménages de Kinshasa, concernant les grossesses antérieures des femmes (y compris celles interrompues) a révélé une hausse de l'incidence de l'avortement en fonction du niveau d'éducation atteint, jusqu'au niveau de scolarité secondaire supérieur ${ }^{21}$. L'incidence était également supérieure parmi les femmes employées dans le « secteur moderne ", par rapport à leurs homologues sans emploi ou employées à leur propre compte, de même que parmi les femmes célibataires par rapport à celles mariées. L'appartenance à certains groupes religieux et ethniques s'est avérée positivement ou négativement associée au fait d'avoir subi un avortement, suivant le groupe spécifique considéré. En 2003, une autre enquête auprès des ménages sur la pratique contraceptive à Kinshasa a 


\section{Distribution en pourcentage des patientes en soins après avortement par caractéristique et rapports de cote estimés pour les associations entre les caractéristiques des patientes et la cause (avortement provoqué ou fausse couche) de l'interruption de la grossesse, Kinshasa, 2016}

\begin{tabular}{|c|c|c|}
\hline CARACTÉRISTIQUE & Distribution en $\%$ & $\begin{array}{l}\text { Rapport des cotes } \\
\text { estimé de l'interruption } \\
\text { de grossesse peut-être } \\
\text { ou certainement provo- } \\
\text { quée vs fausse couche }\end{array}$ \\
\hline \multicolumn{3}{|l|}{ Âge } \\
\hline 15 à 19 ans & 15,7 & 1,42 \\
\hline 20 à 24 ans & 22,9 & 1,19 \\
\hline 25 à 29 ans & 26,7 & 1,09 \\
\hline 30 à 34 ans & 17,3 & 1,07 \\
\hline 35 à 49 ans & 17,4 & réf. \\
\hline \multicolumn{3}{|l|}{ Situation matrimoniale } \\
\hline Célibataire & 59,5 & $13,59 * *$ \\
\hline Mariée & 40,5 & réf. \\
\hline \multicolumn{3}{|l|}{ Éducation } \\
\hline Primaire ou moins & 13,2 & 1,11 \\
\hline Secondaire incomplète & 35,7 & 1,74 \\
\hline Secondaire accomplie & 35,5 & 1,5 \\
\hline Supérieure & 15,7 & réf. \\
\hline \multicolumn{3}{|l|}{$\begin{array}{l}\text { Situation de pauvreté du } \\
\text { ménage }\end{array}$} \\
\hline Pauvre & 40,4 & $1,42 \dagger$ \\
\hline Non pauvre & 59,6 & réf. \\
\hline \multicolumn{3}{|l|}{ Avortements antérieurs } \\
\hline 0 & 72,9 & réf. \\
\hline$\geq 1$ & 27,1 & $2,05^{*}$ \\
\hline \multicolumn{3}{|l|}{ Âge gestationnel } \\
\hline Premier trimestre & 80,9 & réf. \\
\hline Après le premier trimestreł & 19,1 & 0,75 \\
\hline TOTAL & 100 & - \\
\hline
\end{tabular}

NOTES ${ }^{*} p<0,05 . *{ }^{*} p<0,01 . \dagger p<0,10$. $¥$ Cinq cas d'interruption de grossesse déclarée durant le troisième trimestre sont compris dans cette catégorie. N.B.: Sur base des données de 867 patientes en soins après avortement. La catégorie Célibataire comprend les femmes n'ayant jamais été mariées (38\%), celles en concubinage $(18 \%)$ et celles séparées/divorcées $(4 \%)$. Les pourcentages sont calculés avec pondération. réf.=catégorie de référence. Source: référence 6 . révélé une association entre l'âge et l'avortement. Ainsi, les femmes âgées de 15 à 19 ans et de 45 à 49 ans formaient les deux groupes d'âge les plus susceptibles de déclarer avoir interrompu une grossesse ${ }^{24}$. Une étude de 2014 sur des femmes se présentant pour des soins gynécologiques ou obstétricaux d'urgence a révélé que les complications de l'avortement étaient deux fois plus courantes chez les patientes adolescentes que chez celles plus âgées ${ }^{64}$.

Axée sur les femmes demandeuses de soins après avortement, l'Enquête prospective de morbidité de l'Étude de 2016 sur l'avortement à Kinshasa révèle une probabilité d'avortement possible ou certain (plutôt que de fausse couche) plus de 13 fois supérieure parmi les femmes célibataires, par rapport à celles mariées (Tableau 4.2) ${ }^{6}$. L'expérience antérieure de l'avortement y est aussi associée au constat d'avortement possible ou certain: pour les femmes ayant déjà interrompu au moins une grossesse, la probabilité d'obtention de soins après avortement (par opposition à la fausse couche) était deux fois celle associée aux femmes déclarant n'av oir jamais avorté. Enfin, les patientes en soins après avortement issues de ménages pauvres présentaient une probabilité d'avoir certainement ou possiblement avorté de $42 \%$ supérieure à celle de leurs homologues non pauvres.

\section{Contextualisation de l'incidence de I'avortement}

Le choix d'interrompre une grossesse ne dépend bien sûr pas seulement des caractéristiques des femmes, mais est influencé aussi par de nombreux autres facteurs, y compris le contexte juridique, social et culturel dans lequel la décision est prise. La recherche antérieure a démontré que la pratique et la fréquence de l'avortement dans un pays donné n'a pratiquement rien à voir avec sa légalité ou non ${ }^{71}$. Ce statut légal laisse plutôt prédire si l'avortement est plus susceptible de survenir dans des conditions médicalisées ou non. Dans les contextes tels que Kinshasa, où l'avortement est fort limité par la loi, toutes les femmes qui choisissent d'interrompre une grossesse courent le risque d'un avortement non médicalisés9 ${ }^{5}$. Ce risque peut être particulièrement élevé pour les femmes qui n'ont pas les moyens de rechercher et de payer des services sûrs.

Le rang social des femmes et leur exposition aux préjudices sociaux affectent aussi leur besoin et leur décision concernant l'avortement. Étant donné 
la stigmatisation de l'activité sexuelle et de la maternité des femmes célibataires - en particulier dans les sociétés traditionnelles ou conservatrices comme celles souvent observées en RDC - , ces femmes courent un plus grand risque de se faire avorter clandestinement et de souffrir des complications ${ }^{6,39}$. La violence à l'encontre des femmes représente aussi un problème particulièrement important à cet égard. D'après la recherche menée dans la partie orientale du pays, où la violence politique persiste, les femmes qui ont subi des violences sexuelles courent un plus grand risque d'avortement non médicalisé en raison de la stigmatisation des victimes de ces violences, qui peuvent en être "punies » dans les services de soins formels, et du fait aussi de la motivation supérieure à interrompre une grossesse ayant résulté de telles violences ${ }^{39}$.

À Kinshasa, où le type de violence comme arme de guerre observé dans l'est de la RDC est moins répandu, l'association entre la grossesse et le vécu de la violence n'est guère documentée. L'Enquête prospective de morbidité de l'Étude de 2016 sur l'avortement à Kinshasa a cependant interrogé les femmes en soins après avortement sur leur expérience de la violence physique et sexuelle. Plus d'une femme sur cinq (22\%) ont déclaré avoir été frappées, giflées, battues à coups de pied ou autrement agressées physiquement par leur mari ou partenaire (Tableau 4.3) ${ }^{44}$. Parmi ces femmes, plus d'un tiers (34\%) avaient subi des violences physiques perpétrées par leur partenaire pendant la grossesse à propos de laquelle elles étaient venues chercher des soins après avortement. En fait, 37\% des femmes victimes de violences pendant leur grossesse ont indiqué que ces violences avaient contribué à une fausse couche et, pour $25 \%$, qu'elles avaient conduit à un avortement non médicalisé. En outre, 17\% des patientes en soins après avortement ont déclaré avoir déjà subi des rapports sexuels forcés et 30\% d'entre elles ont rapporté que la grossesse à propos de laquelle elles étaient venues se faire soigner était le résultat de ces rapports forcés. Le responsable dans ces cas était le plus souvent le partenaire ou le mari (81\%).

Les observations de l'Étude de 2016 sur l'avortement à Kinshasa concernant l'expérience générale de la violence sont conformes aux données de I'EDS 2013-2014, selon lesquelles 21\% des femmes de la ville avaient subi des violences physiques « parfois ou fréquemment » durant les 12 mois précédant l'enquête et $16 \%$ des femmes avaient
TABLEAU

\section{Distribution en pourcentage des femmes recevant des soins après avortement, en fonction de la violence physique et de rapports sexuels forcés, Kinshasa, 2016}

MESURE

Violence physique* perpétrée par le partenaire ou époux

Distribution en $\%$

A subi une violence physique

\begin{tabular}{ll}
\hline Oui & 22 \\
\hline Non & 78
\end{tabular}

Fréquence de la violence durant les 12 derniers mois $†$

Toujours 3

Souvent

Occasionnellement 6

Jamais 61

30

La violence a eu lieu pendant la grossesse à l'origine de la recherche de SAAt

\begin{tabular}{lc}
\hline Oui & 34 \\
\hline Non & 36 \\
\hline Non déclaré & 30 \\
\hline Changement du niveau de violence par rapport à avant la grossesse & \\
\hline En baisse & 37 \\
\hline Inchangé & 23 \\
\hline En hausse/pire & 14 \\
\hline Ne sait pas & 26 \\
\hline Résultats-santé de cette violence physique $¥, \S^{*} * *$ & 37 \\
\hline Fausse couche & 25 \\
\hline Avortement & 4 \\
\hline Douleur & 4 \\
\hline Hémorragie & 10 \\
\hline Autre & 18 \\
\hline Aucun & 3 \\
\hline
\end{tabular}

\section{Rapports sexuels forcés}

A subi des rapports sexuels forcés§

\begin{tabular}{ll}
\hline Oui & 17 \\
\hline Non & 83
\end{tabular}

La grossesse à l'origine des SAA était le résultat de tels rapports††

\begin{tabular}{ll}
\hline Oui & 30
\end{tabular}

Non 63

Ne sait pas

Responsable §,キ‡

Partenaire/époux

Agent de police 10

Collègue, enseignant/professeur ou responsable inconnu TOTAL

9

NOTES *Frappée giflée, battue à coups de pied ou autrement agressée physiquement par le mari ou partenaire. †Parmi les 180 femmes ayant déjà subi de la violence physique. ¥Parmi les 59 femmes ayant subi de la violence physique pendant la grossesse. §Hors femmes n'ayant pas répondu. **Réponses multiples permises. ††Parmi les 136 femmes ayant déclaré avoir déjà subi des rapports sexuels forcés. ¥¥Parmi les 41 femmes ayant déclaré que la grossesse pour laquelle elles recherchaient des SAA était le résultat de rapports sexuels forcés. N.B.: Analyses basées sur 867 patientes en soins après avortement, sauf indication contraire. Les pourcentages sont calculés avec pondération. SAA=soins après avortement. Source: référence 44. 
subi des violences sexuelles ${ }^{9}$. Il en est d'autant plus important de bien cerner le risque de violence encouru par les femmes et les circonstances qui les mènent à choisir l'avortement.

\section{Opinion des experts sur la réduction de l'avortement non médicalisé et de ses complications en RDC}

Dans le cadre de l'Étude de 2016 sur l'avortement à Kinshasa, les informateurs clés interrogés lors du Sondage des professionnels de la santé et les répondants à l'Enquête dans les formations sanitaires ont été invités à donner leur opinion sur différents aspects de la prestation de l'avortement et des soins après avortement.

Concernant les meilleurs moyens de réduire l'avortement non médicalisé dans la ville et les mesures à prioriser, la grande majorité (87\%) des informateurs clés ayant participé au sondage avaient recommandé l'amélioration de l'information sur la contraception et l'accès aux contraceptifs, tandis que près de la moitié préconisaient la vulgarisation des risques pour la santé posés par l'avortement non médicalisé ${ }^{42}$. La majorité (87\%) s'opposaient à l'idée d'un renforcement de l'application de la loi pénalisant l'avortement, $73 \%$ des informateurs clés estimant plutôt que cette loi devrait être libéralisée.

Presque tous pensaient que l'avortement devrait être légal si la santé physique de la femme est en jeu ${ }^{42}$. Quelque $64 \%$ estimaient qu'il devrait être admis en cas d'anomalie fœtale grave; $50 \%$ I'approuvaient en cas de viol et $36 \%$, en cas d'inceste. Un peu moins du tiers (31\%) étaient favorables à l'avortement en cas d'incapacité intellectuelle de la femme enceinte ou en cas de risque pour sa santé mentale (30\%). L'opinion des répondants à l'Enquête dans les formations sanitaires différait quelque peu de celle des informateurs clés. Quatre cinquièmes ( $81 \%$ ) étaient favorables à l'avortement légal en cas de risque posé à la santé physique de la femme; 54\% en cas d'anomalie fœtale grave et $36 \%$ pour protéger la santé mentale de la femme. Un cinquième des répondants estimaient que l'avortement devrait être admis en cas d'incapacité intellectuelle de la femme ou si la grossesse était le résultat d'un viol ${ }^{72}$. Quinze pour cent seulement estimaient que la loi devrait être changée pour permettre l'avortement dans de plus larges circonstances.
Concernant la prestation des soins après avortement, un peu plus de la moitié des informateurs clés (54\%) pensaient que l'amélioration et le développement d'une infrastructure à cet effet seraient utiles à la réduction des conséquences de l'avortement non médicalisé et $45 \%$ convenaient que l'accroissement du nombre de prestataires qualifiés contribuerait à la réalisation de cet objectif ${ }^{42}$. Parmi les répondants à l'Enquête dans les formations sanitaires, $57 \%$ estimaient que les services pourraient être améliorés en renforçant les capacités techniques, $28 \%$ en assurant une plus grande disponibilité de médicaments et fournitures, $26 \%$ en améliorant et en élargissant l'infrastructure et $15 \%$ en accroissant les effectifs qualifiés ${ }^{72}$. Sept pour cent seulement des répondants jugeaient adéquates les prestations assurées dans leur structure. 


\section{(5) Conclusions et recommandations}

Dans l'adversité, face notamment à plus de deux décennies de conflit armé, les progrès récents de la $\mathrm{RDC}$ en matière de santé sexuelle et reproductive sont prometteurs. L'engagement du pays sur la voie d'une réforme légale propice à un meilleur accès aux services de santé sexuelle et reproductive et sa reconnaissance de l'importance du mariage plus tardif, de l'amélioration de l'accès à l'éducation et de l'autonomisation des femmes contribueront vraisemblablement à un changement positif sur le long terme ${ }^{32}$

Alors même que des mesures sont prises pour mettre en œuvre le Protocole de Maputo et d'autres réformes de politique récentes, beaucoup de Congolaises présentent un besoin urgent de soins de santé sexuelle et reproductive, en particulier sur le plan des services contraceptifs et de soins après avortement. L'Étude de 2016 sur l'avortement à Kinshasa apporte des données à jour concrètes qui mettent en lumière les besoins actuels. Sur les quelque 563100 grossesses survenues dans la ville en $2016,61 \%$ n'étaient pas planifiées, conduisant en fin de compte à un nombre estimé à 146700 avortements $^{5}$.

En raison de la nature fortement restrictive de l'avortement en RDC, la plupart des avortements pratiqués à Kinshasa ne sont pas médicalisés. Beaucoup de femmes souffrent de complications et une grande partie d'entre elles ne reçoivent pas les soins médicaux dont elles ont besoin. Comme à l'échelle mondiale, les effets néfastes pour la santé de l'accès limité aux services de santé sexuelle et reproductive, les soins après avortement compris, à Kinshasa retombent inéquitablement sur les femmes défavorisées, notamment les jeunes et les pauvres.

Les coûts de la défaillance à répondre aux besoins de santé sexuelle et reproductive des femmes sont élevés, tant sur le plan économique qu'en termes de mortalité et de morbidité maternelles.
Les femmes, les familles et la société au sens large en pâtissent. Heureusement, les complications associées à l'avortement non médicalisé sont presque entièrement évitables, de même d'ailleurs que la majorité des grossesses non planifiées qui aboutissent à un avortement. Des mesures doivent donc être prises pour éviter les deux, ainsi que pour améliorer les services existants.

Il n'existe pas de données disponibles sur les coûts de la prestation de soins après avortement à Kinshasa pour le système sanitaire mais, à en juger par les données d'autres contextes nationaux africains, ces coûts sont probablement considérables. Par exemple, au Nigeria, les coûts annuels pour le système sanitaire des soins après avortement en 2007 étaient estimés à 19 millions de dollars ${ }^{73}$. La réponse aux besoins de soins après avortement de toutes les femmes porterait du reste ces coûts pour le système sanitaire de Kinshasa au-delà encore de leur niveau actuel. En Ouganda, le coût annuel total de la prestation de soins après avortement aux niveaux documentés en 2010 a été estimé à 13,9 millions de dollars et la satisfaction du besoin intégral de telles prestations aurait porté ce coût à 20,8 millions de dollars par $a^{74}$. De même, au Rwanda, les soins en 2012 étaient estimés à 1,7 million de dollars par an et la satisfaction du besoin intégral de ces soins aurait porté les coûts à 2,5 millions de dollars par $\mathrm{an}^{75}$.

\section{Recommandations}

Les recommandations énoncées ci-dessous permettraient d'atteindre les objectifs de santé et de développement de la RDC sur le plan de la santé sexuelle et reproductive et de rectifier les résultats de santé reproductive inéquitables et préjudiciables actuellement observés dans le pays. La mise en œuvre de ces recommandations pourrait contribuer grandement à l'amoindrissement du problème de la grossesse non planifiée et de l'avortement non médicalisé à Kinshasa et à l'échelle nationale. 


\section{Poursuivre les efforts récents d'amélioration de I'accès à la contraception et de son adoption parmi les femmes désireuses d'espacer ou de limiter} leurs maternités. II existe de nombreux moyens documentés d'accroître l'accès à la contraception et son adoption, et de soutenir sa pratique continue sur le long terme ${ }^{76}$. L'amélioration de la qualité des services s'est cependant révélée l'un des facteurs les plus propices à l'accroissement de l'adoption de la contraception ${ }^{77-80}$. La prestation de services de contraception de haute qualité met en jeu différents facteurs: assurer que les prestataires possèdent les compétences nécessaires et fassent preuve d'attitudes positives dénuées de jugement, tout en établissant du reste des soins sensibles aux besoins de populations particulières, notamment les femmes jeunes, pauvres, rurales et célibataires. II est aussi essentiel d'offrir à toutes les femmes la méthode de leur choix parmi l'éventail proposé. Ainsi, une étude de données de l'Enquête démographique et de santé de pays d'Afrique, d'Asie et d'Amérique latine a estimé que, pour chaque méthode contraceptive supplémentaire abordable et régulièrement en stock dans des formations sanitaires auxquelles les femmes en quête de contraception peuvent accéder aisément, le taux national de prévalence contraceptive augmente de 5 à 8 points de pourcentage ${ }^{81}$. De même, dans une étude effectuée en RDC, le nombre des couples-années de protection assurées par une formation sanitaire augmente de près de huit pour chaque méthode contraceptive supplémentaire proposée et disponible ${ }^{82}$.

Les interventions génératrices de demande jouent aussi un rôle important pour alerter les femmes de la disponibilité et des avantages de la contraception. Les campagnes médiatiques, les séances d'information individuelle et les transferts monétaires se sont tous avérés propices à l'adoption ${ }^{76}$. Dans une étude menée en 2010 à Butembo, I'une des plus grandes villes de l'est de la RDC, les mères adolescentes n'étaient guère informées sur leurs options de planification familiale traditionnelle ou moderne, mais près d'une sur trois répondantes a demandé à apprendre comment utiliser les méthodes ${ }^{83}$.

Outre les prédicteurs individuels d'adoption, les décideurs politiques de la RDC doivent considérer l'influence des facteurs macroéconomiques locaux et régionaux, des conflits et de l'infrastructure (y compris les voies de transport) dans leur effort d'amélioration de l'accès à la contraception ${ }^{84}$. Le pays est vaste et le progrès sera progressif. Cependant, l'investissement dans l'élargissement et I'amélioration des services de contraception réduira le coût des soins après avortement tout en amoindrissant aussi ceux associés aux soins de santé maternelle et néonatale - libérant en dernière analyse des fonds qui pourront alors être investis davantage dans les systèmes de santé 85,86 .

\section{Effectuer la transition vers les méthodes recom- mandées de prestation de soins après avorte-} ment. À Kinshasa, les prestations de soins après avortement ne sont pas conformes aux meilleures pratiques internationales établies. L'Organisation mondiale de la Santé préconise l'aspiration manuelle intra-utérine (AMIU) pour le traitement de première intention de l'avortement incomplet et elle recommande l'usage du misoprostol en l'absence de prestataire qualifié ${ }^{87}$. Les antibiotiques prophylactiques sont recommandés dans tous les cas sans complications et, pour soulager la douleur, des médicaments anti-inflammatoires non stéroïdiens doivent être proposés à toutes les femmes et administrés immédiatement s'ils sont demandés. Les directives recommandent vivement aussi l'offre de services de contraception à toutes les patientes en soins après avortement.

Pour aligner la prestation des services de Kinshasa sur les normes internationales, il faudra renforcer les capacités du système sanitaire, tant au niveau primaire qu'à celui de référence secondaire. Dans cette attente, les services de soins après avortement peuvent être améliorés progressivement. La dilatation et curetage exige des professionnels de santé hautement qualifiés, un équipement stérile, une salle d'opération et des soins considérables à la patiente. Les frais pour les formations sanitaires comme pour les patientes pourraient être minimisés si les méthodes d'évacuation utérine moins invasives recommandées étaient adoptées ${ }^{88-90}$. De même, la formation du personnel infirmier et des sages-femmes à la prestation sans risque des méthodes AMIU et au misoprostol pour l'avortement incomplet sans complications permettrait d'accroitre les effectifs disponibles pour la prestation de ces méthodes recommandées ${ }^{91}$. Bien que la prise en charge des cas compliqués exige la surveillance de médecins (et parfois de spécialistes), en cas d'infection et d'hémorragie notamment, les professionnels de santé de niveau intermédiaire formés n'en peuvent pas moins gérer la prise en charge initiale.

\footnotetext{
Améliorer l'accès aux services de soins après avortement. L'alignement des services de soins après avortement sur l'orientation internationale améliorerait grandement la qualité des soins. Cela dit, assurer l'accès aux soins exige aussi des services
} 
de qualité qui soient abordables et offerts à proximité des patientes qui en ont besoin ${ }^{92}$. Les Kinoises sont peut-être confrontées à moins d'obstacles géographiques d'accès que leurs homologues hors capitale mais, d'après l'Étude de 2016 sur l'avortement à Kinshasa, la plupart des soins après avortement dispensés dans la ville le sont dans des formations privées et d'ONG, où les services sont vraisemblablement plus chers que dans les formations sanitaires de l'État. Même pour les femmes qui s'adressent au secteur public, il semble que des payements « sous la table » pour ces services soient monnaie courante ${ }^{93}$. Que les femmes choisissent le secteur privé et les ONG ou les formations sanitaires du secteur public, elles encourent vraisemblablement des frais et, comme indiqué ailleurs, les femmes et leur ménage peuvent supporter une part considérable des coûts directs de l'obtention de soins après avortement ${ }^{73}$. Les femmes paient souvent de leur poche une partie des coûts liés à l'avortement dans les pays où l'accès aux services médicalisés est limité ${ }^{73,74,94-96}$ et ces frais ne représentent qu'une partie des coûts des services de santé. Les coûts indirects supplémentaires peuvent inclure la perte de productivité et de revenu, la perturbation de l'éducation des enfants et la détérioration des circonstances économiques au niveau de l'individu et du ménage $\mathrm{e}^{97,98}$.

\section{Résoudre l'inégalité de genre et la violence à} I'encontre des femmes. Des interventions ciblées sur la résolution de l'inégalité de genre et du rôle des femmes en RDC seraient largement bénéfiques, car les femmes contribuent largement au bien-être et à la prospérité de leur famille et de leur communauté ${ }^{99}$, elles rapportent un revenu essentiel et elles exercent une forte influence sur la santé et le niveau d'éducation des enfants ${ }^{97}$. Le vécu de violences perpétrées par un partenaire intime est associé à plus d'un résultat de santé physique et mentale précaire ${ }^{100,101}$. Cela sans compter que les survivantes de la violence sexuelle en RDC font état d'innombrables problèmes de santé et de bien-être psychosocial, y compris les IST et la grossesse non désirée ${ }^{102}$. L'adoption de mesures visant à éliminer toutes les formes de violence à l'encontre des femmes favoriserait grandement la santé, le bien-être et la qualité de la vie des femmes à Kinshasa et en RDC. Réduire les inégalités de genre, soutenir et autonomiser les femmes et résoudre les attitudes dévalorisantes à leur égard ont un potentiel des plus prometteurs, en termes d'amélioration des résultats de santé sexuelle et reproductive, de réduction de la pauvreté et de meilleures perspectives d'avenir pour les générations futures.
Élargir l'accès à l'avortement médicalisé en combattant la stigmatisation et en mettant en œuvre les dispositions du Protocole de Maputo. En changeant le statut légal extrêmement restrictif de I'avortement, on améliorerait considérablement les résultats de santé à Kinshasa, grâce à des interventions plus sûres et plus accessibles. Les défenseurs de la santé des femmes en RDC œuvrent depuis longtemps en faveur de la légalisation de l'avortement, réclamant notamment publiquement sa dépénalisation et faisant plus récemment pression pour la publication du Protocole de Maputo ${ }^{63,103}$. Dans le cadre de l'Étude de 2016 sur l'avortement à Kinshasa (organisée avant la publication du protocole), d'importantes proportions d'informateurs clés et d'administrateurs de formation sanitaire se sont montrés favorables à la libéralisation du code pénal du pays, sans toutefois toujours s'entendre sur les conditions dans lesquelles l'avortement devrait être autorisé.

Globalement, l'opposition socioculturelle à I'avortement a été identifiée comme un obstacle qui empêche non seulement les femmes d'obtenir les soins dont elles ont besoin, mais aussi les prestataires de les dispenser ${ }^{104-107}$. Combattre l'impact de la stigmatisation liée à l'avortement fera partie intégrante de la mise en œuvre des dispositions du Protocole de Maputo. Il faudra sensibiliser les prestataires de soins de santé, les communautés, les décideurs politiques et les autres intervenants. Les ateliers de clarification des valeurs et transformation des attitudes (CVTA) peuvent offrir une ressource précieuse, car ils engagent les participants « dans un processus d'autocritique dans le but de transformer les attitudes et les comportements relatifs à l'avortement de manière favorable aux femmes qui le choisissent ${ }^{108}$. » Une évaluation des sondages pré- et post-atelier CVTA en Asie, en Afrique et en Amérique latine entre 2006 et 2011 a révélé l'amélioration de la connaissance des participants, de leurs attitudes et de leurs comportements attendus à l'égard des soins d'avortement. Le plus fort impact a été identifié parmi les participants qui en savaient le moins et dont les attitudes étaient les plus négatives au départ.

Les données d'impact de la libéralisation des lois nationales sur l'avortement dans d'autres contextes peuvent aussi aider à convaincre les agents de santé et les politiques locaux de la valeur d'un accès élargi en vertu du protocole. En Afrique du Sud, la proportion de la mortalité maternelle imputable à l'avortement non médicalisé dans 
le pays a chuté de $91 \%$ durant les quatre années suivant l'élargissement des indications légales de I'avortement ${ }^{109}$. Cela dit, l'accès à des services médicalisés ne se produit pas du jour au lendemain. La formation et l'équipement des professionnels de la santé et la sensibilisation de la population à l'accès aux services prennent du temps. L'amélioration des soins après avortement restera nécessaire, même après l'élargissement des services médicalisés. L'Éthiopie a changé sa législation fortement restrictive de l'avortement en 2005 et la sensibilisation du public à la disponibilité de l'intervention se poursuit à ce jour. Une évaluation du changement après la réforme légale a révélé, entre 2008 et 2014, une hausse de plus de $39 \%$ du nombre annuel estimé de femmes demandant un avortement légal ${ }^{110}$. Une hausse parallèle a été observée dans la proportion des interventions médicamenteuses, des soins assurés par des agents sanitaires de niveau intermédiaire et des patientes recevant une contraception après avortement. Dans le même temps, le pays a vu plus que doubler le nombre estimé de femmes demandeuses de soins après avortement, sous l'effet combiné peut-être d'une stigmatisation amoindrie et d'un accès accru aux services de soins après avortement.

Renforcer la recherche sur la santé sexuelle et reproductive, y compris sur l'avortement chez les adolescentes. Une recherche accrue serait utile à la compréhension des lacunes des politiques, des services et des pratiques de santé sexuelle et reproductive en RDC. La stratégie nationale à cet égard prévoit notamment l'établissement de centres de jeunesse offrant des services de santé reproductive s'adressant spécifiquement aux jeunes et aux adolescents $^{111}$. La recherche sur la santé sexuelle et reproductive de ces derniers - y compris en ce qui concerne l'avortement dans cette tranche d'âge contribuerait à ces efforts. Il est du reste impératif d'approfondir la base de connaissances relatives aux adolescents, compte tenu en particulier de la jeunesse de la population kinoise.

Il faut aussi documenter les coûts actuels de l'offre de soins après avortement pour le système sanitaire de la RDC. Vraisemblablement élevés, ces coûts pourraient encourager davantage les décideurs politiques à accélérer le rythme de mise en œuvre du Protocole de Maputo.

De plus, au cours de cette mise en œuvre dans le pays, la recherche sur l'impact des mesures prises pour améliorer l'accès à des services d'avortement médicalisé profiterait tant sur le plan intérieur qu'extérieur à la RDC. II n'existe, au mieux, que peu de données sur l'accès des adolescentes à l'avortement et sur leur expérience de la pratique en RDC; les efforts de suivi de l'élargissement de l'accès aux jeunes femmes et aux adolescentes aideraient à combler cette lacune particulière.

\section{Progrès continu}

Voici plus de 30 ans, les chercheurs en RDC posaient que les coûts de l'avortement non médicalisé dans le pays étaient élevés et ils préconisaient comme double stratégie de résolution de ce problème de santé publique l'amélioration de l'accès à la contraception et la libéralisation de la loi sur l'avortement ${ }^{63}$. Les recommandations faites au pays sont étonnamment similaires aujourd'hui. Il existe un besoin d'amélioration de l'infrastructure et de l'accès à des services de santé de qualité en général, mais, en particulier, un besoin de volonté et d'engagement, aussi bien politique que social, à investir dans les services de santé sexuelle et reproductive et à les améliorer. Pour résoudre les niveaux élevés de morbidité et de mortalité parmi les femmes du pays, une attention immédiate est requise. Des mesures doivent être prises pour combattre la stigmatisation de l'avortement et appliquer le Protocole de Maputo aussi rapidement et efficacement que possible. De plus, I'amélioration des services de contraception et de soins après avortement existants contribuera à la réalisation des Objectifs de développement durable, en réduisant les besoins non satisfaits en matière de contraception et partant la mortalité maternelle, entre autres objectifs ${ }^{112}$.

Les développements récents de la politique sur la santé sexuelle et reproductive en RDC offrent une occasion propice à la réalisation d'un changement qui profitera aux femmes, aux familles, aux communautés et, en fin de compte, au pays dans son ensemble. L'élan doit être maintenu si l'on veut en faire une réalité. 


\section{Références}

1. Journal Officiel de la République démocratique du Congo, Code pénal congolais, Décret du 30 janvier 1940 tel que modifié et complété à ce jour, mis à jour au 30 novembre 2004

2. Gouvernement de la République démocratique du Congo, Ordonnance 70-158 du 30 avril 1970 déterminant les règles de la déontologie médicale, 70-158, 1970, https://abortion-policies.srhr.org/documents/countries/03Democratic-Republic-of-the-Congo-Rules-of-MedicalEthics-MoH-1970.pdf.

3. Journal officiel de la République démocratique du Congo, Cabinet du Président de la République, Loi n 06/015 du 12 juin 2006 autorisant l'adhésion de la République démocratique du Congo au Protocole à la Charte africaine des droits de l'homme et des peuples, relatif aux droits de la femme en Afrique, loi ${ }^{\circ}$ 06/015, 2006, http://www.journalofficiel.cd/respubrescente.php?id jo=1369\&lib_parution=Parution\%20sp\%C3\%A9ciale\&lib_ volume $=$ Premi\%C3\%A8re\%20partie\&num_publication_ jo $=\&$ date_publication $=14-03-2018$.

4. Center for Human Rights, University of Pretoria, Maputo Protocol turns 15 ! A call for states to ratify and implement the Protocol- moving from rhetoric to action, 2018, http:// www.chr.up.ac.za/wru-news/832-maputo-protocol-turns15-a-call-for-states-to-ratify-and-implement-the-protocolmoving-from-rhetoric-to-action.

5. Chae $S$ et al., The incidence of induced abortion in Kinshasa, Democratic Republic of Congo, 2016, PLOS ONE, 2017, 12(10):e0184389, doi:10.1371/journal.pone.0184389.

6. Bankole $A$ et al., The severity and management of complications among postabortion patients treated in Kinshasa health facilities, International Perspectives on Sexual and Reproductive Health, 2018, 44(1):1-9, doi:10.1363/44e5618.

7. Rutstein $\mathrm{SO}$ et Johnson $\mathrm{K}$, The DHS wealth index, DHS Comparative Reports, Calverton, MD, USA: ORC Macro, $2004, n^{\circ} 6$.

8. Filmer D et Pritchett LH, Estimating wealth effects without expenditure data-or tears: an application to educational enrollments in states of India, Demography, 2001, 38(1):115-132.

9. Ministère du Plan et Suivi de la Mise en œuvre de la Révolution de la Modernité (MPSMRM) et Ministère de la Santé Publique (MSP), Enquête démographique et de santé en République démocratique du Congo 2013-2014, Kinshasa: MPSMRM et MSP; et Rockville, MD, USA: ICF International, 2014, https://dhsprogram.com/publications/publicationFR300-DHS-Final-Reports.cfm

10. Performance Monitoring and Accountability 2020, PMA2017/Kinshasa, DRC Round 6 Family Planning Brief, 2018, https://www.pma2020.org/sites/default/files/EN-DRCKinshasa-R6-FP-Brief_0.pdf.

11. United Nations Human Settlements Programme, Urbanization and Development: Emerging Futures World Cities Report 2016, Nairobi, Kenya: UN Habitat, 2016.
12. Division de la Population de I'ONU, World Urbanization Prospects: The 2014 Revision, New York: Division de la Population de I'ONU, 2015.

13. Banque mondiale, Population estimates and projections: Democratic Republic of Congo, World Bank DataBank, 2019, https://databank.worldbank.org/ Population-growth-rate-DRC/id/8a4029ee.

14. Fonds monétaire international, Democratic Republic of the Congo: selected issues, IMF Country Report, 2015, No. 15/281, http://elibrary.imf.org/view/IMF002/228829781513590189/22882-9781513590189/228829781513590189.xml.

15. Banque mondiale, Poverty headcount ratio at $\$ 1.90$ a day (2011 PPP) (\% of population), 2019, https://data.worldbank.org/indicator/SI.POV. DDAY?end $=2012 \&$ locations $=C D \&$ start $=2004 \&$ view $=$ map

16. Programme des Nations Unies pour le développement, Human Development Indices and Indicators: 2018 Statistical Update: Congo (Democratic Republic of The), 2018, http:// hdr.undp.org/sites/all/themes/hdr_theme/country-notes/ COD.pdf.

17. Human Rights Watch, DR Congo: chronology, Human Rights Watch, 2009, https://www.hrw.org/ news/2009/08/21/dr-congo-chronology.

18. Kwete $D$ et al., Family planning in the Democratic Republic of the Congo: encouraging momentum, formidable challenges, Global Health: Science and Practice, 2018, 6(1):40-54, doi:10.9745/GHSP-D-17-00346.

19. Peterman A, Palermo T et Bredenkamp C, Estimates and determinants of sexual violence against women in the Democratic Republic of Congo, American Journal of Public Health, 2011, 101(6):1060-1067.

20. Hindin MJ, Kishor S et Ansara DL, Intimate partner violence among couples in $10 \mathrm{DHS}$ countries: predictors and health outcomes, DHS Analytical Studies, Calverton, MD, USA: Macro International Inc., 2008, n 18.

21. Shapiro D et Tambashe BO, The impact of women's employment and education on contraceptive use and abortion in Kinshasa, Zaire, Studies in Family Planning, 1994, 25(2):96-110, doi:10.2307/2138087.

22. Lupwana $K$, Clandestine and risk abortions of female adolescents by self-medication in the outskirts of Kinshasa: vulnerability factors, document présenté à la $6^{\mathrm{e}}$ Conférence africaine sur la population, Ouagadougou, Burkina Faso, du 5 au 9 décembre 2011, https://uaps2011.princeton.edu/ abstracts/110572

23. Paluku LJ et al., Knowledge and attitude of schoolgirls about illegal abortions in Goma, Democratic Republic of Congo, African Journal of Primary Health Care \& Family Medicine, 2010, 2(1), doi:10.4102/phcfm.v2i1.78.

24. Kayembe PK et al., Prevalence and determinants of the use of modern contraceptive methods in Kinshasa, Democratic Republic of Congo, Contraception, 2006, 74(5):400-406, doi:10.1016/j.contraception.2006.06.006.

25. Banque mondiale, GDP per capita (current US\$) Democratic Republic of Congo data, 2019, https://data.worldbank.org/indicator/NY.GDP.PCAP. CD?end $=2016$ \&locations $=C D \&$ start $=1960$.

26. Banque mondiale, GINI index (World Bank estimate) data, 2019, https://data.worldbank.org/indicator/SI.POV.GI $\mathrm{N} I$ ? contextual=default\&locations=CD-ZA-US-NO-KZ. 
27. Organisation mondiale de la Santé, The Abuja Declaration: Ten Years On, 2011, http://www.who.int/ healthsystems/publications/abuja_declaration/en/.

28. USAID, Democratic Republic of the Congo-global health, 2019, https://www.usaid.gov/democratic-republiccongo/global-health.

29. Organisation mondiale de la Santé (OMS), Trends in Maternal Mortality: 1990 to 2015. Estimates by WHO, UNICEF, UNFPA, World Bank Group and the United Nations Population Division, Genève: OMS, 2015, http://www.who. int/reproductivehealth/publications/monitoring/maternalmortality-2015/en/.

30. République démocratique du Congo, Ministère de la Santé publique, Planification familiale, Plan stratégique national à vision multisectorielle (2014-2020), 2014, http:// ec2-54-210-230-186.compute-1.amazonaws.com/wpcontent/uploads/2014/10/DRC_National_Family_Planning_ Plan_French.pdf.

31. Family Planning 2020, New Country Commitments to FP2020: Benin, Democratic Republic of the Congo (DRC), Guinea, Mauritania, and Myanmar, 2013, https://www. familyplanning2020.org/sites/default/files/2013_11-14_ New_Country_Commitments_to_FP2020_Benin_DRC_ Guinea_Mauritania_Myanmar_V2.pdf.

32. Gouvernement de la République démocratique du Congo, Engagements FP 2020, Londres: FP2020, 2017, http://ec2-54-210-230-186.compute-1.amazonaws.com/ wp-content/uploads/2017/08/Engagements-FP2020-DRCRevision-de-2017-2.pdf

33. Ishoso DK et al., Extent of induced abortions and occurrence of complications in Kinshasa, Democratic Republic of the Congo, Reproductive Health, 2019, 16(1), doi:10.1186/s12978-019-0727-4.

34. Moore AM et al., The estimated incidence of induced abortion in Ethiopia, 2014: changes in the provision of services since 2008, International Perspectives on Sexual and Reproductive Health, 2016, 42(3), doi:10.1363/42e1816.

35. Prada $E$ et al., Incidence of induced abortion in Uganda, 2013: new estimates since 2003, PLOS ONE, 2016, 11(11):e0165812, doi:10.1371/journal.pone.0165812.

36. Basinga $\mathrm{P}$ et al., Abortion incidence and postabortion care in Rwanda, Studies in Family Planning, 2012, 43(1):1120.

37. Mohamed SF et al., The estimated incidence of induced abortion in Kenya: a cross-sectional study, BMC Pregnancy and Childbirth, 2015, 15(185), doi:10.1186/s12884-0150621-1.

38. Sedgh $\mathrm{G}$ et al., Estimates of the incidence of induced abortion and consequences of unsafe abortion in Senegal, International Perspectives on Sexual and Reproductive Health, 2015, 41(1):11-19, doi:10.1363/4101115.

39. Burkhardt $\mathrm{G}$ et al., Sexual violence-related pregnancies in eastern Democratic Republic of Congo: a qualitative analysis of access to pregnancy termination services, Conflict and Health, 2016, 10(1), doi:10.1186/s13031-0160097-2.

40. Rouhani $S A$ et al., A quantitative assessment of termination of sexual violence-related pregnancies in eastern Democratic Republic of Congo, Conflict and Health, 2016, 10(1), doi:10.1186/s13031-016-0073-x.
41. Sherris J et al., Misoprostol use in developing countries: results from a multicountry study, International Journal of Gynecology \& Obstetrics, 2005, 88(1):76-81, doi:10.1016/j. ijgo.2004.09.006.

42. Tableaux de données spéciaux de l'Étude de 2016 sur l'avortement à Kinshasa, 2018.

43. OMS, Avortement sécurisé: directives techniques et stratégiques à l'intention des systèmes de santé, Zème édition, 2013, https://www.who.int/reproductivehealth/ publications/unsafe_abortion/9789241548434/fr/.

44. Tableaux de données spéciaux de l'Enquête prospective 2016 de morbidité à Kinshasa, 2018.

45. Johnson-Hanks J, The lesser shame: abortion among educated women in southern Cameroon, Social Science \& Medicine, 2002, 55(8):1337-1349.

46. Boltanski L, La condition foetale: une sociologie de l'engendrement et de l'avortement, Paris: Gallimard Essai, 2004.

47. Théry l, Avortement, engendrement et singularisation des êtres humains, Annales Histoire, Sciences Sociales, 2006, 61(02):481-503, doi:10.1017/S0395264900001384.

48. Figà-Talamanca I et al., Illegal abortion: an attempt to assess its cost to the health services and its incidence in the community, International Journal of Health Services: Planning, Administration, Evaluation, 1986, 16(3):375-389, doi:10.2190/BJ4F-9KJN-MFAL-6X22.

49. Ganatra B et al., Global, regional, and subregional classification of abortions by safety, 2010-14: estimates from a Bayesian hierarchical model, Lancet, 2017, 390(10110):2372-2381, doi:10.1016/S0140-6736(17)31794-4.

50. Singh S et al., Abortion Worldwide 2017: Uneven Progress and Unequal Access, New York: Guttmacher Institute, 2018.

51. Darroch JE, Adding It Up: Investing in Contraception and Maternal and Newborn Health, 2017-Estimation Methodology, New York: Guttmacher Institute, 2018, supplementary table 36, https://www.guttmacher.org/ report/adding-it-up-investing-in-contraception-maternalnewborn-health-2017-methodology.

52. Pande RP et al., Continuing with "... a heavy heart" - consequences of maternal death in rural Kenya, Reproductive Health, 2015, 12(S1), doi:10.1186/1742-475512-S1-S2.

53. Molla $\mathrm{M}$ et al., Impacts of maternal mortality on living children and families: a qualitative study from Butajira, Ethiopia, Reproductive Health, 2015, 12(S1), doi:10.1186/1742-4755-12-S1-S6.

54. Bazile J et al., Intergenerational impacts of maternal mortality: qualitative findings from rural Malawi, Reproductive Health, 2015, 12(S1), doi:10.1186/1742-475512-S1-S1.

55. Knight L et Yamin AE, "Without a mother": caregivers and community members' views about the impacts of maternal mortality on families in KwaZulu-Natal, South Africa, Reproductive Health, 2015, 12(S1), doi:10.1186/17424755-12-S1-S5

56. Kes A et al., The economic burden of maternal mortality on households: evidence from three sub-counties in rural western Kenya, Reproductive Health, 2015, 12(S1), doi:10.1186/1742-4755-12-S1-S3. 
57. Kirigia JM et al., Indirect cost of maternal deaths in the WHO African Region in 2010, BMC Pregnancy and Childbirth, 2014, 14(1), doi:10.1186/1471-2393-14-299.

58. Family Care International (FCl) et al., A Price Too High to Bear: The Costs of Maternal Mortality to Families and Communities, New York: FCl; Washington, DC: International Council for Research on Women; et Nairobi, Kenya: KEMRI/ CDC Research and Public Health Collaborative, 2014.

59. Organisation mondiale de la Santé, Prévention des avortements à risque, 2018, https://www.who.int/fr/newsroom/fact-sheets/detail/preventing-unsafe-abortion.

60. Organisation mondiale de la Santé, Clinical Guidelines for Emergency Treatment of Abortion Complications, 1994, No. WHO/FHE/MSM/94.1, http://www.postabortioncare. org/sites/pac/files/WHO_Clinical_Management_Abortion_ Complications.pdf.

61. Singh S, Darroch JE et Ashford LS, Une vue d'ensemble: coûts et avantages de l'investissement dans la santé sexuelle et reproductive 2014, New York: Guttmacher Institute, 2014.

62. Guttmacher Institute, Soins après avortement à Kinshasa, 2016, infographique, 2018, https://www. guttmacher.org/fr/infographic/2018/soins-apresavortement-kinshasa-2016.

63. Tshibangu K et al., Avortement clandestin, problème de santé publique à Kinshasa (Zaïre), Journal de Gynécologie, Obstétrique et Biologie de la Reproduction, 1984, 13(7):759763.

64. Ishoso DK, Tshefu AK et Coppieters Y, Analysis of induced abortion-related complications in women admitted to referral health facilities in Kinshasa, Democratic Republic of the Congo, PLOS ONE, 2018, 13(8):e0203186, doi:10.1371/journal.pone.0203186.

65. Grossman D et al., Surgical and medical second trimester abortion in South Africa: a cross-sectional study, BMC Health Services Research, 2011, 11(1), doi:10.1186/1472-6963-11-224.

66. Sedgh $\mathrm{G}$ et al., Abortion incidence between 1990 and 2014: global, regional, and subregional levels and trends, Lancet, 2016, 388(10041):258-267, doi:10.1016/S01406736(16)30380-4.

67. Kidman R, Palermo T et Bertrand J, Intimate partner violence, modern contraceptive use and conflict in the Democratic Republic of the Congo, Social Science \& Medicine, 2015, 133:2-10, doi:10.1016/j. socscimed.2015.03.034

68. Muanda MF et al., Barriers to modern contraceptive use in rural areas in DRC, Culture, Health \& Sexuality, 2017, 19(9):1011-1023, doi:10.1080/13691058.2017.1286690.

69. Babazadeh $\mathrm{S}$ et al., Assessing the contraceptive supply environment in Kinshasa, DRC: trend data from PMA2020, Health Policy and Planning, 2018, 33(2):155-162, doi:10.1093/heapol/czx134.

70. Bearak J et al., Global, regional, and subregional trends in unintended pregnancy and its outcomes from 1990 to 2014: estimates from a Bayesian hierarchical model, Lancet Global Health, 2018, 6(4):e380-e389, doi:10.1016/S2214109X(18)30029-9.

71. Sedgh $\mathrm{G}$ et al., Induced abortion: estimated rates and trends worldwide, Lancet, 2007, 370(9595):1338-1345, doi:10.1016/S0140-6736(07)61575-X.
72. Tableaux de données spéciaux de l'Enquête 2016 dans les formations sanitaires de Kinshasa, 2018.

73. Bankole A et al., Estimating the cost of post-abortion care in Nigeria: a case study, dans: Lule E, Singh S et Chowdhury S, eds., Fertility Regulation Behaviors and Their Costs: Contraception and Unintended Pregnancies in Africa and Eastern Europe \& Central Asia, Washington, DC: Banque mondiale, 2007, pp. 65-92.

74. Vlassoff $M$ et al., The health system cost of postabortion care in Uganda, Health Policy and Planning, 2014, 29(1):56-66, doi:10.1093/heapol/czs133.

75. Vlassoff $M$ et al., The health system cost of postabortion care in Rwanda, Health Policy and Planning, 2015, 30(2):223-233, doi:10.1093/heapol/czu006.

76. Mwaikambo $L$ et al., What works in family planning interventions: a systematic review, Studies in Family Planning, 2011, 42(2):67-82, doi:10.1111/j.17284465.2011.00267x.

77. Mensch $B$ et Arends-Kuenning $M$, The impact of the quality of family planning services on contraceptive use in Peru, Studies in Family Planning, 1996, 27(2):59-75.

78. Arends-Kuenning $M$ et Kessy FL, The impact of demand factors, quality of care and access to facilities on contraceptive use in Tanzania, Journal of Biosocial Science, 2007, 39:1-26.

79. Mroz TA et al., Quality, accessibility, and contraceptive use in rural Tanzania, Demography, 1999, 36(1):23-40.

80. Koenig $M$, Hossain $M$ et Whitaker $M$, The influence of quality of care upon contraceptive use in rural Bangladesh, Studies in Family Planning, 1997, 28(4):278-289.

81. Futures Institute, The impact of stock-outs on the use of modern contraception, document présenté à l'assemblée annuelle de la Conférence international sur la planification familiale, Addis Abeba, Éthiopie, 12-13 novembre 2013.

82. Kayembe $P$ et al., Family planning supply environment in Kinshasa, DRC: survey findings and their value in advancing family planning programming, Global Health: Science and Practice, 2015, 3(4):630-645, doi:10.9745/ GHSP-D-15-00298.

83. Mathe JK, Kasonia KK et Maliro AK, Barriers to adoption of family planning among women in eastern Democratic Republic of Congo, African Journal of Reproductive Health, 2011, 15(1):69-77.

84. Kandala NB et al., Disparities in modern contraception use among women in the Democratic Republic of the Congo: a cross-sectional spatial analysis of provincial variations based on household survey data, Journal of Biosocial Science, 2015, 47(3):345-362, doi:10.1017/ S0021932014000212.

85. Johnston HB, Gallo MF et Benson J, Reducing the costs to health systems of unsafe abortion: a comparison of four strategies, Journal of Family Planning and Reproductive Health Care, 2007, 33(4):250-257, doi:10.1783/147118907782101751.

86. Guttmacher Institute, Vue d'ensemble: Investir dans la contraception et la santé maternelle et néonatale en Afrique, Fiche d'information, 2017, https://www.guttmacher.org/fr/ fact-sheet/adding-it-up-contraception-mnh-2017-africa. 
87. OMS, Clinical Practice Handbook for Safe Abortion, Genève: OMS, 2014.

88. American College of Obstetricians and Gynecologists Committee on International Affairs, ACOG committee opinion, 2009, n 427, https://www.acog.org/-/media/ Committee-Opinions/Committee-on-International-Affairs/ co427.pdf?dmc $=1$.

89. Koontz SL et al., Treating incomplete abortion in El Salvador: cost savings with manual vacuum aspiration, Contraception, 2003, 68(5):345-351.

90. You JHS et Chung TKH, Expectant, medical or surgical treatment for spontaneous abortion in first trimester of pregnancy: a cost analysis, Human Reproduction, 2005, 20(10):2873-2878, doi:10.1093/humrep/dei163.

91. OMS, Rôles des agents de santé dans la dispensation des soins liés à l'avortement sécurisé et de la contraception postavortement, Genève: OMS, 2015.

92. Evans DB, Hsu J et Boerma T, Universal health coverage and universal access, Bulletin of the World Health Organization, 2013, $91(8): 546-546$ A, doi:10.2471/ BLT.13.125450.

93. Bertone MP, Lurton $\mathrm{G}$ et Mutombo PB, Investigating the remuneration of health workers in the DR Congo: implications for the health workforce and the health system in a fragile setting, Health Policy and Planning, 2016, 31(9):1143-1151, doi:10.1093/heapol/czv131.

94. Lince-Deroche $\mathrm{N}$ et al., Accessing medical and surgical first-trimester abortion services: women's experiences and costs from an operations research study in KwaZulu-Natal Province, South Africa, Contraception, 2017, 96(2):72-80, doi:10.1016/j.contraception.2017.03.013.

95. Lince-Deroche $\mathrm{N}$ et al., The costs of accessing abortion in South Africa: women's costs associated with secondtrimester abortion services in Western Cape Province, Contraception, 2015, 92(4):339-344, doi:10.1016/j. contraception.2015.06.029.

96. Vlassoff $M$ et al., The health system cost of postabortion care in Ethiopia, International Journal of Gynaecology and Obstetrics, 2012, 118(Suppl. 2):S127-S133, doi:10.1016/S0020-7292(12)60011-3.

97. Sundaram $A$ et al., Documenting the individual- and household-level cost of unsafe abortion in Uganda, International Perspectives on Sexual and Reproductive Health, 2013, 39(04):174-184, doi:10.1363/3917413.

98. Vlassoff $M$ et al., Estimates of health care system costs of unsafe abortion in Africa and Latin America, International Perspectives on Sexual and Reproductive Health, 2009, 35(3):114-121, doi:10.1363/ipsrh.35.114.09.

99. Banque mondiale, Women: The Future of Africa, Washington, DC: Banque mondiale, 2018.

100. Ellsberg $\mathrm{M}$ et al., Intimate partner violence and women's physical and mental health in the WHO multicountry study on women's health and domestic violence: an observational study, Lancet, 2008, 371(9619):1165-1172, doi:10.1016/S0140-6736(08)60522-X.

101. Gibbs A, Dunkle K et Jewkes R, Emotional and economic intimate partner violence as key drivers of depression and suicidal ideation: a cross-sectional study among young women in informal settlements in South Africa, PLOS ONE, 2018, 13(4):e0194885, doi:10.1371/ journal.pone.0194885.
102. Bartels $S$ et al., Surviving sexual violence in eastern Democratic Republic of Congo, Journal of International Women's Studies, 2010, 11(4):37-49.

103. Kalonda JCO, Sexual violence in Congo-Kinshasa: necessity of decriminalizing abortion, Revue médicale de Bruxelles, 2011, 33:482-486.

104. Culwell KR et Hurwitz $M$, Addressing barriers to safe abortion, International Journal of Gynecology \& Obstetrics, 2013, 121(S1):S16-S19, doi:10.1016/j.ijgo.2013.02.003.

105. Rehnström Loi $U$ et al., Health care providers' perceptions of and attitudes towards induced abortions in sub-Saharan Africa and Southeast Asia: a systematic literature review of qualitative and quantitative data, $B M C$ Public Health, 2015, 15(1):139, doi:10.1186/s12889-0151502-2.

106. Payne $\mathrm{CM}$ et al., Why women are dying from unsafe abortion: narratives of Ghanaian abortion providers, African Journal of Reproductive Health, 2013, 17(2):118-128.

107. Mohamed D, Diamond-Smith $\mathrm{N}$ et Njunguru J, Stigma and agency: exploring young Kenyan women's experiences with abortion stigma and individual agency, Reproductive Health Matters, 2018, 26(52):128-137, doi:10.1080/0968808 0.2018 .1492285 .

108. Turner KL et al., Values clarification workshops to improve abortion knowledge, attitudes and intentions: a pre-post assessment in 12 countries, Reproductive Health, 2018, 15(1):40, doi:10.1186/s12978-018-0480-0.

109. Jewkes $R$ et Rees $H$, Dramatic decline in abortion mortality due to the Choice on Termination of Pregnancy Act, South African Medical Journal, 2005, 95(4):250-250.

110. Gebrehiwot $Y$ et al., Changes in morbidity and abortion care in Ethiopia after legal reform: national results from 2008 and 2014, International Perspectives on Sexual and Reproductive Health, 2016, 42(3):121-130, doi:10.1363/42e1916.

111. Evidence to Action, Government of DRC announces creation of first national platform for engaging Congolese youth in AYSRH decision-making, press release, 2017, https://www.e2aproject.org/news-item/government-drcannounces-creation-first-national-platform-engagingcongolese-youth-aysrh-decision-making/.

112. Nations Unies, Sustainable Development Goals Knowledge Platform, Health and population, sans date, https://sustainabledevelopment.un.org/topics/ healthandpopulation. 
Grossesses non planifiées et avortements à Kinshasa (République Démocratique du Congo) : Défis et progress est disponible en ligne. Pour télécharger, visitez https://www.guttmacher.org/fr/report/ unintended-pregnancy-abortion-in-Kinshasa-drc.

(C)2019 Guttmacher Institute, société sans but lucratif vouée à la promotion de la santé sexuelle et reproductive partout dans le monde, à travers la recherche, l'analyse des politiques et la sensibilisation du public. Tous droits, y compris de traduction dans d'autres langues, réservés en vertu de la Convention universelle sur le droit d'auteur, de la Convention de Berne pour la protection des œuvres littéraires et artistiques et des Conventions interet panaméricaines sur le droit d'auteur (Mexico et Buenos Aires). Des dérogations peuvent être accordées pour les droits de traduction de l'information contenue dans ce rapport.

ISBN: 978-1-934387-21-4

Indication de source suggérée: Lince-Deroche N et al., Grossesses non planifiées et avortements à Kinshasa (République Démocratique du Congo) : Défis et progrès, New York: Guttmacher Institute, 2019, https://www.guttmacher.org/fr/report/ unintended-pregnancy-abortion-in-Kinshasa-drc. https://doi.org/10.1363/2019.30887
Guttmacher Institute 125 Maiden Lane New York, NY 10038 USA

Téléphone: +1-212-248-1111

Fax: +1-212-248-1951

info@guttmacher.org

\section{www.guttmacher.org}

Département des Sciences de la Population et du Développement Université de Kinshasa BP 176

Kinshasa XI

République Démocratique du Congo Téléphone: +243 (81) 4963860

dspd.unikin@unikin.ac.cd

crispin.mabika@unikin.ac.cd

\section{www.unikin.ac.cd}

École de Santé Publique

Université de Kinshasa

BP 11850

Kinshasa I

République Démocratique du Congo

Téléphone: +243 (99) 9945183

espsec_unikin@yahoo.fr

www.unikin.ac.cd 
